\title{
POINTWISE CONVERGENCE OF THE SCHRÖDINGER FLOW
}

\author{
E. COMPAAN, R. LUCÀ, AND G. STAFFILANI
}

\begin{abstract}
In this paper we address the question of the pointwise almost everywhere limit of nonlinear Schrödinger flows to the initial data, in both the continuous and the periodic settings. Then we show how, in some cases, certain smoothing effects for the non-homogeneous part of the solution can be used to upgrade to a uniform convergence to zero of this part, and we discuss the sharpness of the results obtained. We also use randomization techniques to prove that with much less regularity of the initial data, both in continuous and the periodic settings, almost surely one obtains uniform convergence of the nonlinear solution to the initial data, hence showing how more generic results can be obtained.
\end{abstract}

There may be differences between this version and the published version. You are advised to consult the publisher's version:

E. Compaan, R. Lucà and G. Staffilani, Pointwise Convergence of the Schrödinger Flow, International Mathematics Research Notices, Volume 2021, Issue 1, January 2021, Pages 596-647, https://doi.org/10.1093/imrn/rnaa036.

\section{Contents}

1. Introduction

1.1. Acknowledgements

1.2. Notations and terminology

2. Preliminaries $\quad 7$

3. Deterministic Results 11

3.1. The Linear Schrödinger Equation on $\mathbb{T}^{d} \quad 11$

3.2. The NLS Equation on $\mathbb{T}^{d}$ and $\mathbb{R}^{d}$ (Theorem 1.1) 13

4. Probabilistic Results 18

4.1. The Linear Schrödinger Equation on $\mathbb{T}^{d}$ with Random Data 18

4.2. The Linear Schrödinger Equation on $\mathbb{R}^{d}$ with Random Data 21

4.3. The Cubic NLS Equation on $\mathbb{T}^{d}(d=1,2)$ with Random Data (Theorem 1.3)

4.4. The Cubic NLS Equation on $\mathbb{R}^{d}(d=1,2)$ with Random Data

(Theorem 1.5) 36

References

37

Date: January 4, 2022.

Key words and phrases. Schrödinger equation, maximal estimates, smoothing estimates, random data. 


\section{INTRODUCTION}

In this work, we are concerned with the question of almost everywhere convergence of solutions to certain nonlinear Schrödinger equations (NLS) to initial data. More precisely, let $u(x, t)$ be a solution to

$$
\left\{\begin{array}{l}
i \partial_{t} u+\Delta u=\mathcal{N}(u), \quad x \in \mathbb{T}^{d} \text { or } \mathbb{R}^{d}, \\
u(x, 0)=f(x),
\end{array}\right.
$$

where $\mathbb{T}:=\mathbb{R} / 2 \pi \mathbb{Z}$ and $\mathcal{N}$ is a power type nonlinearity. If $f \in H^{s}$, for what $s$ do we have that $u(x, t) \rightarrow f(x)$ as $t \rightarrow 0$ for (Lebesgue) almost every $x$ ?

In the linear Euclidean setting, namely $\mathcal{N}=0$ and $x \in \mathbb{R}^{d}$, this question was first posed by Carleson [11], who showed that almost everywhere (a.e.) convergence holds for $f \in H^{\frac{1}{4}}(\mathbb{R})$. Dahlberg-Kenig [15] showed that this one dimensional result is sharp; in fact they proved that $s \geq \frac{1}{4}$ is a necessary condition for a.e. convergence on $\mathbb{R}^{d}, d \geq 1$. Since then, the higher dimensional problem has been studied by many authors $[14,10,39,47,3,32,45,46,43,27,6,28,16,29,20]$. Recently, Bourgain [7] proved that $s \geq \frac{d}{2(d+1)}$ is a necessary condition for a.e. pointwise convergence to the data (see also [30] for an alternative counterexample). This has been proved to be sharp, up to the endpoint, by Du-Guth-Li [19] in the $\mathbb{R}^{2}$ case, and by DuZhang [18] in higher dimensions.

In the linear periodic setting, namely $\mathcal{N}=0$ and $x \in \mathbb{T}^{d}$, much less is known. The only result appears to be that of Mouya-Vega [33] when $d=1$, (sufficiency of $s>\frac{1}{3}$ and necessity of $s \geq \frac{1}{4}$ ), which method of proof, based on Strichartz estimates, has been extended to higher dimensions by Wang-C. Zhang [48]. Together with recent improvements in periodic Strichartz estimates [8], one can show that $s>\frac{d}{d+2}$ is a sufficient condition for almost everywhere convergence to initial data ${ }^{1}$. We refer to Section 3.1 for more details. In Section 3.1 we also show that almost everywhere convergence fails when $s<\frac{d}{2(d+1)},{ }^{2}$ see Proposition 3.2. At the moment, in the periodic case almost sure convergence when $s \in\left[\frac{d}{2(d+1)}, \frac{d}{d+2}\right]$ remains an open question.

In the first part of this paper we extend these results to the nonlinear setting. Hereafter $\Omega$ denotes either $\mathbb{T}$ or $\mathbb{R}$. We define

$$
s_{\Omega^{d}}:= \begin{cases}\frac{d}{d+2} & \text { if } \Omega=\mathbb{T}, \\ \frac{d}{2(d+1)} & \text { if } \Omega=\mathbb{R} .\end{cases}
$$

Summarizing the results mentioned above, one has

$$
\lim _{t \rightarrow 0} e^{i t \Delta} f(x)=f(x) \text { for a.e. } x \in \Omega^{d}
$$

for all $f \in H^{s}\left(\Omega^{d}\right)$ with $s>s_{\Omega^{d}}$. If $\Omega^{d}=\mathbb{R}$ we only need $s \geq s_{\mathbb{R}}=\frac{1}{4}$.

In the following theorem we prove that a similar result is true for solutions to NLS with power nonlinearities.

\footnotetext{
${ }^{1}$ Although in this paper we only consider rational tori, this particular result holds for any torus since it is based on Strichartz estimates, now available for any torus thanks to [8].

${ }^{2}$ This follows adapting the non periodic counterexamples to the periodic setting and still works if we consider irrational tori.
} 
Theorem 1.1. Let $\mathcal{N}(z)= \pm|z|^{p-1} z$ with $p \geq 3$. If $f \in H^{s}\left(\Omega^{d}\right)$ with

$$
s>\max \left(s_{\Omega^{d}}, \frac{d}{2}-\frac{2}{p-1}\right),
$$

and $u$ is the corresponding solution to (1), then

$$
\lim _{t \rightarrow 0} u(x, t)=f(x) \quad \text { for a.e. } x \in \Omega^{d} .
$$

If $\Omega^{d}=\mathbb{R}$ and $p<9$ we can relax the condition $s>s_{\mathbb{R}}=\frac{1}{4}$ to $s \geq \frac{1}{4}$. Moreover if we consider the cubic equation $(p=3)$ and $d=1$ or $\Omega^{d}=\mathbb{R}^{2}$ we have for $s>\frac{d}{6}$

$$
\lim _{t \rightarrow 0} u(x, t)-e^{i t \Delta} f(x)=0 \quad \text { for every } x \in \Omega^{d}
$$

and the convergence is uniform with respect to the $x$ variable ${ }^{3}$, namely

$$
\lim _{t \rightarrow 0} \sup _{x \in \Omega^{d}}\left|u(x, t)-e^{i t \Delta} f(x)\right|=0 .
$$

Remark 1.2. The result is sharp in the following sense. The conditions $p \geq 3$ and

$$
s>\max \left(0, \frac{d}{2}-\frac{2}{p-1}\right),
$$

ensure that the flow is locally well defined, in fact $s_{c}:=\frac{d}{2}-\frac{2}{p-1}$ is the critical exponent. The extra assumption $s>s_{\Omega^{d}}$ ensures that the linear part $e^{i t \Delta} f$ of the flow converges pointwise a.e. to the initial datum $f$. This condition is sharp if $\Omega=\mathbb{R}$ (modulo endpoints when $d \geq 2$ ) and we do not expect improved convergence to the data when we introduce a nonlinearity. Moreover by the proof of Theorem 1.1 it will be clear that any improvement of the exponent $s_{\Omega^{d}}$ into the linear setting would provide an analogous improvement of Theorem 1.1 as well. More precisely, if we define

$$
s_{\Omega^{d}}^{*}:=\inf \left\{s: \lim _{t \rightarrow 0} e^{i t \Delta} f(x)=f(x) \quad \text { for a.e. } x \in \Omega^{d}, \quad \forall f \in H^{s}\left(\Omega^{d}\right)\right\},
$$

we can replace the assumption (3) by

$$
s>s_{\Omega^{d}}^{*} \quad \text { and } \quad s>\frac{d}{2}-\frac{2}{p-1},
$$

and we can relax $s>s_{\Omega^{d}}^{*}$ to $s \geq s_{\Omega^{d}}^{*}$ if the inf in (6) is a min.

In the second part of this paper we adopt a different approach than the purely deterministic one we presented in the first part that culminated in Theorem 1.1. More precisely we consider the linear and the cubic NLS and we show that actually uniform convergence to the data is generically true for initial data which are less smooth than the data postulated in Theorem 1.1. In the periodic setting, we consider

$$
f^{\omega}(x)=\sum_{n \in \mathbb{Z}^{d}} \frac{g_{n}^{\omega}}{\langle n\rangle^{\frac{d}{2}+\alpha}} e^{i n \cdot x}, \quad \alpha>0,
$$

where $g_{n}^{\omega}$ are independent (complex) standard Gaussian variables and we define $\langle\cdot\rangle=\left(1+|\cdot|^{2}\right)^{\frac{1}{2}}$. We will need the following facts, proved in Section 4.1. Fix $t \in \mathbb{R}$. Then $e^{i t \Delta} f^{\omega}(x)$ belongs to $\bigcap_{s<\alpha} H^{s}\left(\mathbb{T}^{d}\right) \mathbb{P}$-almost surely. Thus we are working at

\footnotetext{
${ }^{3} \mathrm{~A}$ proof of (5) in the case $d=1$ is in $[21,13]$. Here we extend the result to $\Omega^{d}=\mathbb{R}^{2}$.
} 
the $H^{\alpha-}$ level. Moreover, $e^{i t \Delta} f^{\omega}$ is $\mathbb{P}$-almost surely a continuous function of the $x$ variable, where $\mathbb{P}$ is the law of the sequence $\left\{g_{n}^{\omega}\right\}_{n \in \mathbb{Z}}$.

In the following statement we consider the Wick ordered cubic NLS in $\mathbb{T}^{d}, d=$ 1,2. Namely equation (1) with nonlinearity

$$
\mathcal{N}(u):= \pm u\left(|u|^{2}-2 \mu\right), \quad \mu:=f_{\mathbb{T}^{d}}|u(x, t)|^{2} d x
$$

Since once we fix the initial datum $f \in L^{2}$, solutions to this equation are related to that of the cubic NLS by multiplication with a factor $e^{i 2 \mu t}$, the study of pointwise convergence of (9) turns out to be equivalent to that of the cubic NLS.

Theorem 1.3. Let $f^{\omega}$ be defined in (8) for $\alpha>0$. Then one has $\mathbb{P}$-almost surely the following. For all $t \in \mathbb{R}$ the free solution $e^{i t \Delta} f^{\omega}$ belongs to $\bigcap_{s<\alpha} H^{s}\left(\mathbb{T}^{d}\right)$ and is continuous in the $x$ variable. Moreover

$$
\lim _{t \rightarrow 0} e^{i t \Delta} f^{\omega}(x)=f^{\omega}(x) \quad \text { for every } x \in \mathbb{T}^{d} .
$$

and the convergence is uniform in the $x$ variable. Let $d=1,2$ and let $u$ be the solution to the Wick ordered cubic NLS (9) with random initial data $f^{\omega}$ as above. Again, $\mathbb{P}$-almost surely one has

$$
\lim _{t \rightarrow 0} u(x, t)=f^{\omega}(x) \quad \text { for a.e. } x \in \mathbb{T}^{d} .
$$

Furthermore, if $\alpha>\frac{d-1}{2}$, then

$$
\lim _{t \rightarrow 0} u(x, t)-e^{i t \Delta} f^{\omega}(x)=0 \quad \text { for every } x \in \mathbb{T}^{d} .
$$

and the convergence is uniform in the $x$ variable.

Remark 1.4. Notice that if $d=1$ combining (10) and (12) we get in fact a stronger convergence statement than (11), namely the convergence occurs at any $x$ and uniformly $(\mathbb{P}$-almost surely). If $d=2$ the combination of (10) and (12) gives this stronger convergence result only for data that are in $H^{\frac{1}{2}+}\left(\mathbb{T}^{2}\right)$, while by (11) we see that a.e. convergence occurs for initial data that are merely in $H^{0+}\left(\mathbb{T}^{2}\right)$ (P-almost surely).

We also obtain results for randomized initial data on Euclidean spaces. We use an integer tiling-type randomization, of the type introduced in [49, 31, 2]. To begin, we construct a partition of unity on $\mathbb{R}^{d}$. Let $\eta$ be a smooth cut-off of the unit interval. Specifically, let $\eta: \mathbb{R}^{d} \rightarrow[0,1]$ be a smooth function such that $\operatorname{supp} \eta \subset\{\xi:|\xi| \leq 2\}$ and $\eta(\xi)=1$ for all $|\xi| \leq 1$. Then for $n \in \mathbb{Z}^{d}$, define

$$
\psi_{n}(\xi)=\frac{\eta(\xi-n)}{\sum_{\ell \in \mathbb{Z}^{d}} \eta(\xi-\ell)} .
$$

Observe that $\psi_{n}$ is smooth function supported on $\{\xi:|\xi-n| \leq 2\}$ and we have $\sum_{n} \psi_{n}(\xi)=1$ for all $\xi$.

Fix $f \in H^{s}\left(\mathbb{R}^{d}\right)$ with $s>0$. We construct a randomization $f^{\omega}$ of $f$ as follows. Let $g_{n}^{\omega}$ be a collection of independent (complex) standard Gaussian variables and define $f^{\omega}$ by

$$
\widehat{f^{\omega}}(\xi)=\sum_{n \in \mathbb{Z}^{d}} g_{n}^{\omega} \psi_{n}(\xi) \widehat{f}(\xi) .
$$

This randomization satisfies analogous properties to the one described above in the periodic setting. If $f^{\omega} \in H^{s}\left(\mathbb{R}^{d}\right)$ then $e^{i t \Delta} f^{\omega}$ is in $H^{s}$ and is a continuous function 
of the $x$ variable $\mathbb{P}$-almost surely, for all $t \in \mathbb{R}$; we refer to Section 4.2 for more details. In order to compare (14) with (8) it is convenient to look at the Fourier transform of (8), that is

$$
\widehat{f^{\omega}}(n)=\frac{g_{n}^{\omega}}{\langle n\rangle^{\frac{d}{2}+\alpha}}
$$

and remember that $\psi_{n}(\xi)$ is a bump function of a neighborhood of $\xi=n$ and that a function with Fourier coefficients $\lesssim\langle n\rangle^{-\frac{d}{2}-\alpha}$ Belongs to $H^{\alpha-}$. We then have the following

Theorem 1.5. Fix $f \in H^{s}\left(\mathbb{R}^{d}\right)$ with $s>0$. Let $f^{\omega}$ be a randomization of $f$ as defined in (14). Then one has $\mathbb{P}$-almost surely the following. For all $t \in \mathbb{R}$ the free solution $e^{i t \Delta} f^{\omega}$ belongs to $H^{s}\left(\mathbb{R}^{d}\right)$ and that is continuous in the $x$ variable. Moreover

$$
\lim _{t \rightarrow 0} e^{i t \Delta} f^{\omega}(x)=f^{\omega}(x) \quad \text { for every } x \in \mathbb{R}^{d}
$$

and the convergence is uniform in the $x$ variable. Let then $u$ be the solution to the cubic NLS with initial data $f^{\omega}$. If $d=1,2$ and $s>\frac{d}{6(d+1)}$, again $\mathbb{P}$-almost surely

$$
\lim _{t \rightarrow 0} u(x, t)=f^{\omega}(x) \quad \text { for almost every } x \in \mathbb{R}^{d} .
$$

Remark 1.6. Both the randomization procedures described do not improve smoothness; see for example Remark 1.2 in [9] and the introduction of [31].

Remark 1.7. The statements (10), (16) are still true, with same proof, as explained in Sections 4.1, 4.2, if we consider random Fourier series drawn from distribution with sufficiently strong decay properties. In fact, the argument we present works for independent $g_{n}^{\omega}$ drawn from a sequence of centered sub Gaussian random variables with unitary variance.

Remark 1.8. The randomization precedures give convergence $e^{i t \Delta} f^{\omega} \rightarrow f^{\omega}$ for any $x \in \Omega^{d}$ (and uniformly) $\mathbb{P}$-almost surely; see (10), (16). In the deterministic case one has convergence at any $x \in \mathbb{R}^{d}$ (and uniformly) only for $s>d / 2$. In fact when $s \leq d / 2$ it make sense to consider a refined version of the a.e. convergence problem, which consists into determine the (worst possible) Hausdorff dimension of the set where the convergence to $H^{s}\left(\mathbb{R}^{d}\right)$ initial data fails. This problem, introduced in [38], has been solved in [1] when $s \in\left[\frac{d}{4}, \frac{d}{2}\right]$. In the range $s \in\left(\frac{d}{2(d+1)}, \frac{d}{4}\right)$, where the problem is still open, the best positive and negative results to date are in [18] and $[29,30]$, respectively.

We now give a brief description of our methods of proof for the three main theorems listed above.

To prove (4) in Theorem 1.1 we consider (smooth) approximations of the solutions of NLS obtained by truncating (1) on the first $N$ Fourier modes. Since for this class of solutions we have pointwise convergence to the initial data, we are able to rewrite the convergence problem as an $L_{x, l o c}^{2}$ bound for a suitable maximal function, adapted to the nonlinear setting; see Proposition 3.3. It is worth mentioning that (already in the linear setting) the maximal function approach is the most powerful tool to study a.e. pointwise convergence to the initial data. In order to obtain a 
good enough bound, in Proposition 3.3 we embed the restriction space $X_{\delta}^{s, \frac{1}{2}+}$ into the space

$$
\left\{F(x, t):\left\|\sup _{0 \leq t \leq \delta}|F(x, t)|\right\|_{L_{x, l o c}^{2}}<\infty\right\},
$$

for $s>s_{\Omega^{d}}$; see Proposition $2.2([0, \delta], \delta>0$ will be the local existence time). This embedding allows us to use Strichartz estimates to conclude the proof; see Section 3.2.

For the cubic NLS we can prove stronger results, taking advantage of the algebraic structure of the nonlinearity $\mathcal{N}(z)= \pm|z|^{2} z$. A first example of this phenomenon is already in the statement (5). Let us consider $x \in \mathbb{R}^{2}$ to fix the notations (similar observations can be made if $x \in \Omega$ ). Since for $s>s_{\mathbb{R}^{2}}=\frac{1}{3}$ one has $e^{i t \Delta} f(x) \rightarrow f(x)$ as $t \rightarrow 0$ for a.e. $x \in \mathbb{R}^{2}$, we see that (5) is clearly stronger than (4). In fact, we can show that for any $t \in \mathbb{R}$, the function

$$
x \in \mathbb{R}^{2} \rightarrow u(x, t)-e^{i t \Delta} f(x) \in \mathbb{C}
$$

is continuous. Moreover the map

$$
t \in \mathbb{R} \rightarrow u(x, t)-e^{i t \Delta} f \in C_{x}\left(\mathbb{R}^{2}\right) \quad\left(\text { with the } \sup _{x \in \mathbb{R}^{2}}\right. \text { norm) }
$$

is also continuous. This stronger convergence result is a consequence of a smoothing effect associated to the cubic nonlinearity on $\mathbb{R}^{2}$, that we prove in Corollary 2.6. A similar smoothing effect has been noted in $1 d$ in both the periodic ${ }^{4}$ [21] and nonperiodic [13] settings: the nonlinearity turns out to be $\sigma$-smoother than the initial datum in $H^{s}$, with $\sigma<\max \left(2 s, \frac{1}{2}\right)$. We strengthen and extend this smoothing effect on $\mathbb{R}^{d}, d=1,2$ to $\sigma<\max (2 s, 1)$; see Corollary 2.6. Using these facts and the Sobolev embedding $H^{\frac{1}{2}+}(\Omega) \hookrightarrow L^{\infty}(\Omega)$, we see that the (1d analog of) property (18) is satisfied by initial data in $H^{s}(\Omega)$ with $s>\frac{1}{6}$. On the other hand, in [37] (see also [30]) it has been observed that for any $s<\frac{1}{4}$ there are initial data such that $\lim \sup _{t \rightarrow 0}\left|e^{i t \Delta} f(x)\right|=\infty$ for $x$ in a set of strictly positive measure. This construction, done for $\Omega=\mathbb{R}$, is based on the Dahlberg-Kenig counterexample and can be repeated also in the periodic setting. Combining this with the above mentioned smoothing effect of the $1 d$ cubic NLS, it is immediate to see that the convergence statement (4) fails for $s \in\left(\frac{1}{6}, \frac{1}{4}\right)$ for the one-dimensional cubic NLS $(p=3)$. However, as observed in Remark 1.2, we expect (4) to fail for any $s<s_{\Omega^{d}}^{*}$ and for any $p \geq 3$.

The proofs of Theorem 1.3 and Theorem 1.5 rely upon a combination of the following two facts. First, the randomization improves the integrability of the randomized function. This allows us to deduce uniform convergence of the linear propagator $e^{i t \Delta} f^{\omega}$ to the initial data $f^{\omega}, \mathbb{P}$-almost surely; see Propositions 4.1 and 4.5. Second, we deduce a smoothing effect associated to the cubic nonlinearity. This allows us to control the nonlinear (Duhamel) contribution $u(x, t)-e^{i t \Delta} f^{\omega}$. While in the Euclidean case (Theorem 1.5), we use the deterministic smoothing effect given in Corollary 2.6, in the periodic case (Theorem 1.3) the proof is much more involved. In fact, we follow the argument of Bourgain in [5] and we start with the Wick-reordering of the nonlinearity. Then using more probabilistic arguments and Jarnick's theorem (namely counting lattice points on convex archs), as in [5], we obtain in Proposition 4.6 a precise quantification of the amount of smoothing

\footnotetext{
${ }^{4}$ In fact in the periodic setting one has to consider the Wick ordered equation; see the paragraph before Theorem 1.3 and Section 4.3 for details.
} 
(that in this case happens $\mathbb{P}$-almost surely). In our argument a quantification of the smoothing is necessary because we need to be sure that the nonlinear (Duhamel) contribution sits in $X_{\delta}^{s, \frac{1}{2}+}$ with $s>s_{\mathbb{T}^{d}}$ (we consider $d=1,2$ ), so that we can conclude the proof by implementing techniques from Theorem 1.1.

1.1. Acknowledgements. E. Compaan is supported by NSF MSPRF 1704865. R. Lucà is supported by the ERC grant 676675 FLIRT, by BERC 2018-2021, by BCAM Severo Ochoa SEV-2017-0718 and IHAIP project PGC2018-094528-B-I00 (AEI/FEDER, UE). G. Staffilani is supported by NSF grants DMS 1462401 and DMS 1764403. The authors thank Chenjie Fan for useful discussions on Propositions 4.1 and 4.5 and the referees for their useful comments.

1.2. Notations and terminology. For a fixed $p \in \mathbb{R}$ we often use the notation $p+:=p+\varepsilon, p-:=p-\varepsilon$, where $\varepsilon$ is any sufficiently small strictly positive real number. When in the same inequality we have two such quantities we use the following notation to compare them. We write $p+\cdots+:=p+\varepsilon \cdot($ number of + ), $p-\cdots-:=p-\varepsilon \cdot($ number of - ). We will use $C>0$ to denote several constants depending only on fixed parameters, like for instance the dimension $d$. The value of $C$ may clearly differ from line to line. Let $A, B>0$. We may write $A \lesssim B$ if $A \leq C B$ when $C>0$ is such a constant. We write $A \gtrsim B$ if $B \lesssim A$ and $A \sim B$ when $A \lesssim B$ and $A \gtrsim B$. We write $A \ll B$ if $A \leq c B$ for $c>0$ sufficiently small (and depending only on fixed parameters) and $A \gg B$ if $B \ll A$. We denote $A \wedge B:=\min (A, B)$ and $A \vee B:=\max (A, B)$. We refer to the following inequality

$$
\left\|D^{s} P_{N} f\right\|_{L^{q}} \lesssim N^{s+\frac{d}{p}-\frac{d}{q}}\left\|P_{N} f\right\|_{L^{p}}, \quad 1 \leq p \leq q \leq \infty,
$$

simply as Bernstein inequality. Here $P_{N}$ is the frequency projection on the annulus $\xi \sim N$.

\section{Preliminaries}

Let us recall that $\Omega$ denotes either $\mathbb{T}$ or $\mathbb{R}$. We denote by $B_{\rho}$ a ball of radius $\rho>0$ centered at a generic point of $\Omega^{d}$ or $\mathbb{Z}^{d}$. The following Strichartz estimates are the main tool to study the nonlinear Schrödinger flow:

$$
\left\|e^{i t \Delta} f(x)\right\|_{L_{x, t}^{p}\left(\Omega^{d+1}\right)} \lesssim N^{\frac{d}{2}-\frac{d+2}{p}+}\|f\|_{L_{x}^{2}\left(\Omega^{d}\right)}, \quad p \geq 2\left(\frac{d+2}{d}\right), \quad \text { supp } \widehat{f} \subseteq B_{N} .
$$

These estimates were proved in [41] for $\Omega=\mathbb{R}$ and in [8] for $\Omega=\mathbb{T}$. The additional factor $N^{0+}$ is removable except when $\Omega=\mathbb{T}$ and $p=2\left(\frac{d+2}{d}\right)$; see $[4,26]$ and the references therein. However, we never use this finer information.

Hereafter $\delta \in(0,1]$, sometimes we will restrict to sufficiently small values of $\delta$. The main tool used in the study of the a.e. pointwise convergence of solutions to linear Scrödinger equation to the initial data is the following maximal estimate

$$
\left\|\sup _{0 \leq t \leq \delta}\left|e^{i t \Delta} f(x)\right|\right\|_{L_{x}^{2}\left(B_{1}\right)} \lesssim\|f\|_{H_{x}^{s}\left(\Omega^{d}\right)} .
$$

The validity of this estimate is equivalent to the fact that $e^{i t \Delta} f(x) \rightarrow f(x)$ as $t \rightarrow 0$ for almost every (with respect to the Lebesgue measure) $x \in B_{1}$. One implication of this statement is elementary; the other is a consequence of the Stein-Nikišhin maximal principle [40,35]. Inequality (20) holds for all $s>s_{\Omega^{d}}$ where $s_{\Omega^{d}}$ is defined in (2); see the introduction and the forthcoming Proposition 3.1. 
The main result of this section (Lemma 2.2) is that given a function

$$
F:(x, t) \in \Omega^{d} \times \mathbb{R} \rightarrow F(x, t) \in \mathbb{C}
$$

we can bound the $L_{x}^{2}\left(B_{1}\right)$ norm of the associated maximal function $\sup _{0 \leq t \leq \delta}|F(x, t)|$ with an appropriate $X_{\delta}^{s, b}$ norm of $F$; see also [4]. This embedding is used to obtain pointwise convergence results for solutions to the nonlinear Schrödinger equation.

We recall that

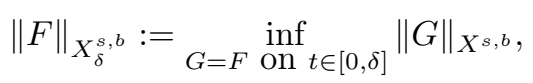

where

$$
\begin{aligned}
\|F\|_{X^{s, b}}^{2} & :=\int_{\mathbb{R}} \sum_{n \in \mathbb{Z}^{d}}\left\langle\tau+|n|^{2}\right\rangle^{2 b}\langle n\rangle^{2 s}|\widehat{F}(n, \tau)|^{2} d \tau \quad \text { if } \quad \Omega=\mathbb{T}, \\
\|F\|_{X^{s, b}}^{2}: & :=\int_{\mathbb{R}} \int_{\mathbb{R}^{d}}\left\langle\tau+|\xi|^{2}\right\rangle^{2 b}\langle\xi\rangle^{2 s}|\widehat{F}(\xi, \tau)|^{2} d \xi d \tau \quad \text { if } \quad \Omega=\mathbb{R},
\end{aligned}
$$

$\langle\cdot\rangle:=\left(1+|\cdot|^{2}\right)^{\frac{1}{2}}$, and $\widehat{F}$ is the space-time Fourier transform of $F$.

The next lemma shows how to embed $X_{\delta}^{s, b}, b>\frac{1}{2}$, into several functional spaces. The proof can be found in [44, Lemma 2.9], in the case $\Omega=\mathbb{R}$. The argument adapts to $\Omega=\mathbb{T}$.

Lemma 2.1. Let $b>\frac{1}{2}$ and let $Y$ be a Banach space of functions

$$
F:(x, t) \in \Omega^{d} \times \mathbb{R} \rightarrow F(x, t) \in \mathbb{C} .
$$

Let $\alpha \in \mathbb{R}$. Assume

$$
\left\|e^{i \alpha t} e^{i t \Delta} f(x)\right\|_{Y} \leq C\|f\|_{H^{s}\left(\Omega^{d}\right)},
$$

with a constant $C>0$ uniform over $\alpha \in \mathbb{R}$. Then

$$
\|F\|_{Y} \leq C\|F\|_{X^{s, b}} .
$$

Using Lemma 2.1 with

$$
\|F\|_{Y}=\left\|\sup _{0 \leq t \leq \delta}|F(x, t)|\right\|_{L_{x}^{2}\left(B_{1}\right)}
$$

and the fact that the maximal estimate (20) hold for $s>s_{\Omega^{d}}\left(s \geq \frac{1}{4}\right.$ if $\left.\Omega^{d}=\mathbb{R}\right)$, we have the following

Lemma 2.2. Let $b>\frac{1}{2}$ and $s>s_{\Omega^{d}}$ defined in (2). We have

$$
\left\|\sup _{0 \leq t \leq \delta}|F(x, t)|\right\|_{L_{x}^{2}\left(\Omega^{d}\right)} \lesssim\|F\|_{X_{\delta}^{s, b}} .
$$

If $\Omega^{d}=\mathbb{R}$ we can relax $s>s_{\mathbb{R}}=\frac{1}{4}$ to $s \geq \frac{1}{4}$.

We also recall several useful estimates, repeatedly used in the paper, that can be obtained using the Strichartz estimates (19) and Lemma 2.1. Let $\mathrm{P}_{N}$ be the frequency projection into the annulus of size $N$, namely $\{N / 2<|\xi| \leq N\}$. Let $\mathrm{P}_{A}$ be the frequency projection into the set $A$. We denote by $Q_{N}$ a (frequency) cube of side length $N$, centered at any point. Then in the statement of the lemma below we use $\Gamma_{N}$ to denote either $Q_{N}$ or the (frequency) annulus of size $N$. Thus $\mathrm{P}_{\Gamma_{N}}$ is either $\mathrm{P}_{Q_{N}}$ or $\mathrm{P}_{N}$. 
Lemma 2.3. Let $p \geq 2\left(\frac{d+2}{d}\right)$. Then

$$
\begin{aligned}
&\left\|\mathrm{P}_{\Gamma_{N}} F\right\|_{L_{x, t}^{p}\left(\Omega^{d+1}\right)} \lesssim N^{\frac{d}{2}-\frac{d+2}{p}-s+}\left\|\mathrm{P}_{\Gamma_{N}} F\right\|_{X^{s, \frac{1}{2}+}}, \\
&\left\|\mathrm{P}_{\Gamma_{N}} F\right\|_{X^{0,-\frac{1}{2}++}} \lesssim N^{0+}\left\|\mathrm{P}_{\Gamma_{N}} F\right\|_{L_{x, t}^{2 \frac{d+2}{d+4}}{ }_{\left(\Omega^{d+1}\right)}} .
\end{aligned}
$$

Let also $s>\frac{d}{2}-\frac{d+2}{p}$, then

$$
\|F\|_{L_{x, t}^{2 \frac{d+2}{d-2 s}-}{ }_{\left(\Omega^{d+1}\right)}} \lesssim\|F\|_{X^{s, \frac{1}{2}+}} .
$$

Proof. By dyadic decomposition in frequency, summing a geometric series, and then using Plancherel, we see that (19) implies

$$
\left\|e^{i t \Delta} f\right\|_{L_{x, t}^{p}\left(\Omega^{d+1}\right)} \lesssim\|f\|_{H^{\frac{d}{2}-\frac{d+2}{p}+}{ }_{\left(\Omega^{d}\right)}} .
$$

This and Lemma 2.1 imply

$$
\|F\|_{L_{x, t}^{p}\left(\Omega^{d+1}\right)} \lesssim\|F\|_{X^{\frac{d}{2}-\frac{d+2}{p}+, \frac{1}{2}+}} \cdot
$$

Now the estimate (23) is an immediate consequence of (26). Letting $p=2\left(\frac{d+2}{d}\right)$ in (23) and interpolating it with $\|\cdot\|_{X^{0,0}}=\|\cdot\|_{L_{x, t}^{2}\left(\Omega^{d+1}\right)}$ we get

$$
\left\|\mathrm{P}_{\Gamma_{N}} F\right\|_{L_{x, t}^{2 \frac{d+2}{d}-}\left(\Omega^{d+1}\right)} \lesssim N^{0+}\left\|\mathrm{P}_{\Gamma_{N}} F\right\|_{X^{0, \frac{1}{2}--}} .
$$

Dualizing this yields (24). The estimate (25) follows from (23) by taking $p=$ $2\left(\frac{d+2}{d-2 s}\right)-$ and $\mathrm{P}_{\Gamma_{N}}=\mathrm{P}_{N}$, after again performing a dyadic frequency decomposition, summing a geometric series, and using Plancherel.

We also recall some well known properties of the $X^{s, b}$ spaces that are repeatedly used in the paper; see for example [25]. Hereafter $\eta$ is a smooth cut-off of the unit interval.

Lemma 2.4. Let $s \in \mathbb{R}$. Then

$$
\begin{gathered}
\left\|\eta(t) e^{i t \Delta} f(x)\right\|_{X^{s, \frac{1}{2}+}} \lesssim\|f\|_{H^{s}\left(\Omega^{d}\right)}, \\
\left\|\eta(t) \int_{0}^{t} e^{i\left(t-t^{\prime}\right) \Delta} F\left(\cdot, t^{\prime}\right) d t^{\prime}\right\|_{X^{s, \frac{1}{2}+}} \lesssim\|F\|_{X^{s,-\frac{1}{2}+}}, \\
\|F\|_{X_{\delta}^{s,-\frac{1}{2}+}} \lesssim \delta^{0+}\|F\|_{X_{\delta}^{s,-\frac{1}{2}++}} .
\end{gathered}
$$

We end this section with a bilinear estimate in the space $\mathbb{R}^{2}$ and in $\mathbb{R}$. We first prove the result in $\mathbb{R}^{2}$. This is the harder case, and a proof has already appeared in [12]; we report it below for completeness. The analogous result in $\mathbb{R}$ is easier and can be proved with similar techniques. These blinear estimates are used to obtain smoothing results for the nonlinear (Duhamel) part of the solution.

We start with some notation. For dyadic numbers $M_{0}, M_{1}, M_{2}$ we set $M_{*}=$ $\min \left(M_{0}, M_{1}, M_{2}\right)$ and $M^{*}=\max \left(M_{0}, M_{1}, M_{2}\right)$. We use the notation $f \chi_{\{|\mu| \sim M\}}=$ : $f_{M}$ for the restriction to a dyadic annulus. We then define

$$
\int_{\tau_{0}+\tau_{1}+\tau_{2}=0, \mu_{0}+\mu_{1}+\mu_{2}=0}=: \int_{*}
$$


and

$$
C_{\mp}(f ; g, h)=\int_{*} f\left(\mu_{0}, \tau_{0}\right) \frac{g\left(\mu_{1}, \tau_{1}\right)}{\left(1+\left.\left|\tau_{1}-\right| \mu_{1}\right|^{2}\right)^{b}} \frac{h\left(\mu_{2}, \tau_{2}\right)}{\left(1+\left.\left|\tau_{2} \pm\right| \mu_{2}\right|^{2}\right)^{b}} d \tau_{1} d \tau_{2} d \mu_{1} d \mu_{2},
$$

with $b>\frac{1}{2}$.

Lemma 2.5 ([12, Lemma 1$])$. Assume we are in $\mathbb{R}^{2}$. The following estimates hold

$$
\begin{gathered}
\left|C_{+}\left(f_{M_{0}} ; g_{M_{1}}, h_{M_{2}}\right)\right| \lesssim\left(\frac{M_{*}}{M^{*}}\right)^{\frac{1}{2}}\left\|f_{M_{0}}\right\|_{L^{2}}\left\|g_{M_{1}}\right\|_{L^{2}}\left\|h_{M_{2}}\right\|_{L^{2}}, \\
\left|C_{-}\left(f_{M_{0}} ; g_{M_{1}}, h_{M_{2}}\right)\right| \lesssim\left(\frac{M_{1} \wedge M_{2}}{M_{1} \vee M_{2}}\right)^{\frac{1}{2}}\left\|f_{M_{0}}\right\|_{L^{2}}\left\|g_{M_{1}}\right\|_{L^{2}}\left\|h_{M_{2}}\right\|_{L^{2}} .
\end{gathered}
$$

We then have the following corollary.

Corollary 2.6. Assume we are in $\mathbb{R}^{d}, d=1,2, b>b^{\prime}>\frac{1}{2}$ and $s \geq 0$. Then if $\sigma<\min (2 s, 1)$ we have

$$
\left\||u|^{2} u\right\|_{X^{s+\sigma, b^{\prime}-1}} \lesssim\|u\|_{X^{s, b}}^{3}
$$

Proof. We describe the proof in dimension $d=2$, which is the hardest case. At the end of the proof we comment on the case $d=1$. Using a dyadic decomposition and duality we need to estimate for $v \in X^{0,1-b^{\prime}}$

$$
M_{0}^{\sigma+s}\left|\int u_{M_{1}} \bar{u}_{M_{2}} u_{M_{3}} \bar{v}_{M_{0}} d x d t\right| .
$$

Without loss of generality we can assume that $M_{1} \geq M_{3}$. Also, below we present the calculation as if $v \in X^{0, b}$, but we can adjust this by possibly losing an $\varepsilon$ on the highest frequency, and this can be done by assuming that $b^{\prime}<b$. We then consider two cases, when $M_{1} \geq M_{2}$ or when $M_{1} \leq M_{2}$. In the first case the most dangerous situation is when $M_{0} \sim M_{1}$. In this case we have

$$
M_{0}^{\sigma+s}\left|\int u_{M_{1}} \bar{u}_{M_{2}} u_{M_{3}} \bar{v}_{M_{0}} d x d t\right| \lesssim M_{0}^{\sigma+s}\left\|u_{M_{1}} u_{M_{3}}\right\|_{L^{2}}\left\|\bar{u}_{M_{2}} \bar{v}_{M_{0}}\right\|_{L^{2}} .
$$

After renormalizing and using the lemma above with $C_{+}$we can continue with

$$
\begin{aligned}
& \lesssim M_{0}^{\sigma+s} M_{1}^{-\frac{1}{2}} M_{3}^{\frac{1}{2}} M_{0}^{-\frac{1}{2}} M_{2}^{\frac{1}{2}}\left\|u_{M_{1}}\right\|_{X^{0, b}}\left\|u_{M_{2}}\right\|_{X^{0, b}}\left\|u_{M_{3}}\right\|_{X^{0, b}}\left\|v_{M_{0}}\right\|_{X^{0, b}} \\
& \lesssim M_{0}^{\sigma-1} M_{3}^{\frac{1}{2}-s} M_{2}^{\frac{1}{2}-s}\left\|u_{M_{1}}\right\|_{X^{s, b}}\left\|u_{M_{2}}\right\|_{X^{s, b}}\left\|u_{M_{3}}\right\|_{X^{s, b}}\left\|v_{M_{0}}\right\|_{X^{0, b}} .
\end{aligned}
$$

If $s \leq \frac{1}{2}$ then we need $\sigma-2 s<0$ and hence $\sigma<2 s$. If $s>\frac{1}{2}$ then we need $\sigma<1$. Assume now that $M_{1} \leq M_{2}$ and that again $M_{0} \sim M_{2}$. With a similar argument we estimate

$$
M_{0}^{\sigma+s}\left|\int u_{M_{1}} \bar{u}_{M_{2}} u_{M_{3}} \bar{v}_{M_{0}} d x d t\right| \lesssim M_{0}^{\sigma+s}\left\|u_{M_{1}} \bar{u}_{M_{2}}\right\|_{L^{2}}\left\|\bar{v}_{M_{0}} u_{M_{3}}\right\|_{L^{2}} .
$$

After renormalizing and using the lemma above with $C_{-}$we obtain a similar result.

The previous argument (and Lemma 2.5) adapts to the case $d=1$; see also [25]. Alternatively one can deduce them from the case $d=2$ using Lemmata 3.1 and 3.6 in [42]. 


\section{Deterministic Results}

\subsection{The Linear Schrödinger Equation on $\mathbb{T}^{d}$.}

In this section we focus on maximal estimates of the linear Schödinger flow

$$
\left\|\sup _{0 \leq t \leq 1}\left|e^{i t \Delta} f(x)\right|\right\|_{L_{x}^{2}\left(\mathbb{T}^{d}\right)} \lesssim\|f(x)\|_{H_{x}^{s}\left(\mathbb{T}^{d}\right)} .
$$

As mentioned in Section 2, it is standard that this estimate implies pointwise convergence $e^{i t \Delta} f(x) \rightarrow f(x)$ as $t \rightarrow 0$ for almost every $x \in \mathbb{T}^{d}$. The problem of identifying the minimal regularity $s$ for which (31) holds is still open. The following result has been proved in [33] when $d=1$ and in [48] when $d \geq 2$. The proof is based on Strichartz estimates. However, comparing with [48], the exponent in the next proposition is better for $d \geq 3$ due to the use of the optimal periodic Strichartz estimates from [8]. Thus, we recall the proof for the sake of completeness.

Proposition 3.1. The inequality (31) holds for all $s>\frac{d}{d+2}$.

Proof. By dyadic frequency decomposition (here $N \in 2^{\mathbb{N}}$ ) it is sufficient to prove that

$$
\left\|\sup _{0 \leq t \leq 1}\left|e^{i t \Delta} \mathrm{P}_{N} f\right|\right\|_{L^{2}\left(\mathbb{T}^{d}\right)} \lesssim N^{s}\left\|\mathrm{P}_{N} f\right\|_{L^{2}\left(\mathbb{T}^{d}\right)}
$$

holds for all $s>\frac{d}{d+2}$; we recall that $\mathrm{P}_{N}$ is the frequency projection into the annulus of size $N$.

We actually prove the stronger estimate

$$
\left\|\sup _{0 \leq t \leq 1}\left|e^{i t \Delta} \mathrm{P}_{N} f\right|\right\|_{L^{2 \frac{d+2}{d}\left(\mathbb{T}^{d}\right)}} \lesssim N^{\frac{d}{d+2}+}\left\|\mathrm{P}_{N} f\right\|_{L^{2}\left(\mathbb{T}^{d}\right)}
$$

We use the following inequality (see [27]), that holds by the Fundamental Theorem of Calculus and Hölder's inequality,

$$
\sup _{0 \leq t \leq 1}|\phi(t)| \lesssim|\phi(0)|+\alpha^{\frac{1}{p}-1}\left\|\partial_{t} \phi(t)\right\|_{L_{t}^{p}([0,1])}+\alpha^{\frac{1}{p}}\|\phi(t)\|_{L_{t}^{p}([0,1])},
$$

with $\phi(t)=e^{i t \Delta} \mathrm{P}_{N} f(x)$. The parameter $\alpha>0$ will be chosen later in such a way as to equalize the second and third term on the right hand side of (34). Since $\partial_{t} e^{i t \Delta} \mathrm{P}_{N} f(x)=i \Delta e^{i t \Delta} \mathrm{P}_{N} f(x)$ we obtain

$$
\begin{aligned}
& \sup _{0 \leq t \leq 1}\left|e^{i t \Delta} \mathrm{P}_{N} f(x)\right| \\
& \lesssim\left|\mathrm{P}_{N} f(x)\right|+\alpha^{\frac{1}{p}-1}\left\|\Delta e^{i t \Delta} \mathrm{P}_{N} f(x)\right\|_{L_{t}^{p}([0,1])}+\alpha^{\frac{1}{p}}\left\|e^{i t \Delta} \mathrm{P}_{N} f(x)\right\|_{L_{t}^{p}([0,1])} \\
& \lesssim\left|\mathrm{P}_{N} f(x)\right|+\alpha^{\frac{1}{p}-1} N^{2}\left\|e^{i t \Delta} \mathrm{P}_{N} f(x)\right\|_{L_{t}^{p}([0,1])}+\alpha^{\frac{1}{p}}\left\|e^{i t \Delta} \mathrm{P}_{N} f(x)\right\|_{L_{t}^{p}([0,1])}
\end{aligned}
$$

Specifying $\alpha=N^{2}$ and taking the $L_{x}^{p}\left(\mathbb{T}^{d}\right)$ norm of (35) we arrive at

$$
\begin{aligned}
\left\|\sup _{0 \leq t \leq 1}\left|e^{i t \Delta} \mathrm{P}_{N} f\right|\right\|_{L^{p}\left(\mathbb{T}^{d}\right)} \lesssim\left\|\mathrm{P}_{N} f\right\|_{L^{p}\left(\mathbb{T}^{d}\right)}+N^{\frac{2}{p}}\left\|e^{i t \Delta} \mathrm{P}_{N} f\right\|_{L^{p}\left(\mathbb{T}^{d} \times[0,1]\right)} \\
\lesssim N^{\frac{d}{2}-\frac{d}{p}}\left\|\mathrm{P}_{N} f\right\|_{L^{2}\left(\mathbb{T}^{d}\right)}+N^{\frac{2}{p}}\left\|e^{i t \Delta} \mathrm{P}_{N} f\right\|_{L^{p}\left(\mathbb{T}^{d} \times[0,1]\right)}
\end{aligned}
$$

where in the second estimate we used Bernstein's inequality. Letting $p=2\left(\frac{d+2}{d}\right)$ and using the Strichartz estimates (19) we obtain (33). 
In the proof of Proposition 3.1 one can replace $\mathbb{T}^{d}$ by $\mathbb{R}^{d}$. However, in the latter case more powerful techniques are available.

We now analyze the sharpness of the maximal estimate (31). We exhibit a counterexample which shows that in fact the maximal estimate (31) fails for sufficiently rough data. Proposition 3.2 below follows adapting the non periodic counterexamples to the periodic setting. Remarkably, it has been proved in [33] that in the case $d=1$ there are different counterexamples which lead to the necessity of $s>1 / 4$, exploiting the quadratic Gauss summation. Here we give another one dimensional counterexample for the necessity of $s>1 / 4$, based on the Galilean invariance of the Schrödinger equation. It is worth to mention that the quadratic Gauss summation approach is instead related to the pseudoconformal invariance. The equivalence between these symmetries in the convergence problem has been already observed when the (linear) problem was settled on $\mathbb{R}^{d}$, comparing the counterexmples in $[16,29]$ and $[7,30]$.

Proposition 3.2. The inequality (31) fails for all $s<\frac{d}{2(d+1)}$.

Proof. One can adapt the non periodic counterexamples. The maximal inequality $(31)$ is disproved using a family of initial data frequency supported on a ball of radius $R>1$ (letting then $R \rightarrow \infty$ ). The time interval at which the $R$-th member $f_{R}$ of this family attains the $\sup _{t}\left|e^{i t \Delta} f_{R}(x)\right|$ is contained in $[0,1 / R]$, for all $R>1$. Since in this time/frequency region there is essentially no difference between periodic and non periodic solutions, the procedure is straightforward. If $d=1$ one can prove this statement in different ways (see [33]). Here we also give an alternative proof. Let $\kappa \in\left(0, \frac{1}{2}\right), N \gg 1$ and $D=\left\lfloor N^{1-\kappa}\right\rfloor$. We first focus on the family of initial data

$$
f(x)=\sum_{\substack{k \in \mathbb{Z} \\|k| \leq N / D}} e^{i D k x}
$$

and the corresponding solutions

$$
e^{i t \Delta} f(x)=\sum_{|k| \leq N / D} e^{i\left(D k x-D^{2}|k|^{2} t\right)}
$$

Notice that

$$
\left|e^{i t \Delta} f(x)\right| \sim \sum_{|k| \leq N / D} 1 \sim N / D \sim N^{\kappa} \quad \text { if } \quad(x, t) \in X \times T
$$

where

$$
X:=D^{-1} \mathbb{Z}+B\left(0, \frac{1}{10 N}\right), \quad T:=D^{-2} \mathbb{Z} .
$$

This is because we can write elements $t \in T$ as $\left\lfloor N^{1-\kappa}\right\rfloor^{-2} \tau$ with $\tau \in \mathbb{Z}$, so that

$$
D^{2}|k|^{2} t=D^{2}|k|^{2} D^{-2} \tau=|k|^{2} \tau \in \mathbb{Z}
$$

and we can write elements $x \in X$ as $x=D^{-1} \ell+\varepsilon$, with $\ell \in \mathbb{Z}, \varepsilon \in \mathbb{R}$ with $|\varepsilon| \leq \frac{1}{10 N}$, so that

$$
D k x=D k\left(D^{-1} \ell+\varepsilon\right)=k \ell+D k \varepsilon \in \mathbb{Z}+B(0, D|k||\varepsilon|)
$$

and

We consider instead the modulated initial data

$$
D|k||\varepsilon| \leq N^{1-\kappa} N^{\kappa} \frac{1}{10 N} \leq \frac{1}{10} .
$$

$$
\tilde{f}(x)=e^{i x} f(x)
$$


where $f$ is chosen as in (37). The corresponding solutions are

$$
e^{i t \Delta} \tilde{f}(x)=e^{i(x-t)}\left(e^{i t \Delta} f\right)(x-2 t) .
$$

Thus, recalling (39), we have

$$
\sup _{0 \leq t \leq 1 / D}\left|e^{i t \Delta} \tilde{f}(x)\right| \gtrsim N^{\kappa} \quad \text { if } x \in \bigcup_{t \in T \cap[0,1 / D]} X+t,
$$

Since the set $\bigcup_{t \in T \cap[0,1 / D]} X+t$ is equidistributed in $\mathbb{T}$, its measure is of order $\left.1 \wedge\left(D^{2} N^{-1}\right) \sim 1 \wedge N^{1-2 \kappa}\right)$, the first factor being the cardinality of $T \cap[0,1 / D]$, the second the cardinality of the small balls of $X \cap \mathbb{T}$, and the last the volumes of these balls. Thus the set $\bigcup_{t \in T \cap[0,1 / D]} X+t$ has full measure for all large $N$ since we assume $\kappa<1 / 2$. By (40) and noting that

$$
\|\tilde{f}\|_{L^{2}(\mathbb{T})}=\|f\|_{L^{2}(\mathbb{T})} \sim(N / D)^{1 / 2} \sim N^{\frac{\kappa}{2}},
$$

the maximal inequality (31) implies that

$$
N^{\kappa} \lesssim N^{s} N^{\frac{\kappa}{2}}
$$

Letting $N \rightarrow \infty$ this leads to a contradiction if $s<\frac{\kappa}{2}$. Since we have restricted to $\kappa<\frac{1}{2}$ we have disproved the inequality (31) for all $s<\frac{1}{4}$.

\subsection{The NLS Equation on $\mathbb{T}^{d}$ and $\mathbb{R}^{d}$ (Theorem 1.1).}

In this section we prove Theorem 1.1. Thus we focus on the NLS equation (1) on $\Omega^{d}$, where $\Omega=\mathbb{R}$ or $\Omega=\mathbb{T}$. The nonlinearity is $\mathcal{N}(z)= \pm|z|^{p-1} z$ with $p \geq 3$. We focus on initial data in $H^{s}\left(\Omega^{d}\right)$ with

$$
s>\max \left(0, \frac{d}{2}-\frac{2}{p-1}\right) .
$$

For such data the flow is locally well defined; see [24, 44] for the non-periodic case and [4] for the periodic one. Let $\Phi_{t}^{N}$ be the flow associated to the truncated NLS equation

$$
i \partial_{t} \Phi_{t}^{N} f+\Delta \Phi_{t}^{N} f=\mathrm{P}_{\leq N} \mathcal{N}\left(\Phi_{t}^{N} f\right),
$$

with initial datum $\Phi_{0}^{N} f:=\mathrm{P}_{\leq N} f$. As usual $\mathrm{P}_{\leq N}$ denotes the frequency projection on the ball of radius $N$ centered in the origin. We write $\Phi_{t} f:=\Phi_{t}^{\infty} f$ for the flow of the NLS equation with initial datum $f=\mathrm{P}_{\infty} f$. We also denote $\mathrm{P}_{>N}:=\mathrm{P}_{\infty}-\mathrm{P}_{\leq N}$ and as alreday mentioned $\mathrm{P}_{N}:=\mathrm{P}_{\leq N}-\mathrm{P}_{\leq N / 2}$.

The following maximal estimate ensures a.e. pointwise convergence to the data. This is the nonlinear analog of the maximal estimate (20).

Proposition 3.3. Let $f \in L^{2}\left(\Omega^{d}\right)$ be such that

$$
\lim _{N \rightarrow \infty}\left\|\sup _{0 \leq t \leq \delta}\left|\Phi_{t} f(x)-\Phi_{t}^{N} f(x)\right|\right\|_{L_{x}^{2}\left(B_{1}\right)}=0
$$

for any $B_{1} \subset \Omega^{d}$. Then $\Phi_{t} f(x) \rightarrow f(x)$ as $t \rightarrow 0$ for almost every $x \in \Omega^{d}$.

From the proof it will be clear that in (43) we can replace the $L^{2}$ norm with the (smaller) $L^{1}$ norm. However is usually convenient to work in $L^{2}$ setting. 
Proof. To prove Proposition 3.3 we decompose the difference as follows:

$$
\left|\Phi_{t} f(x)-f(x)\right| \leq\left|\Phi_{t} f(x)-\Phi_{t}^{N} f(x)\right|+\left|\Phi_{t}^{N} f(x)-\mathrm{P}_{\leq N} f(x)\right|+\left|\mathrm{P}_{>N} f(x)\right|
$$

and pass to the limit $t \rightarrow 0$. The second term on the right hand side is zero. In fact, since $\mathrm{P}_{\leq N} f$ is smooth once has immediately that

$$
\lim _{t \rightarrow 0} \Phi_{t}^{N} f(x)=\mathrm{P}_{\leq N} f(x),
$$

for all $x \in \Omega^{d}$. So we arrive at ${ }^{5}$

$$
\limsup _{t \rightarrow 0}\left|\Phi_{t} f-f\right| \leq \limsup _{t \rightarrow 0}\left|\Phi_{t} f-\Phi_{t}^{N} f\right|+\left|\mathrm{P}_{>N} f\right| .
$$

Let $\lambda>0$. Using the Chebyshev inequality

$$
\begin{aligned}
\mid\left\{x \in B_{1}: \limsup _{t \rightarrow 0}\left|\Phi_{t} f-f\right|\right. & >\lambda\}|\leq|\left\{x \in B_{1}: \sup _{0 \leq t \leq \delta}\left|\Phi_{t} f-\Phi_{t}^{N} f\right|>\lambda / 2\right\} \mid \\
+\left|\left\{x \in B_{1}:\left|\mathrm{P}_{>N} f\right|>\lambda / 2\right\}\right| & \\
& \lesssim \lambda^{-2}\left(\left\|\sup _{0 \leq t \leq \delta}\left|\Phi_{t} f-\Phi_{t}^{N} f\right|\right\|_{L^{2}\left(B_{1}\right)}^{2}+\left\|\mathrm{P}_{>N} f\right\|_{L^{2}\left(B_{1}\right)}^{2}\right)
\end{aligned}
$$

where $|\cdot|$ is the Lebesgue measure. On the other hand we have $\left\|\mathrm{P}_{>N} f\right\|_{L^{2}\left(\Omega^{d}\right)} \rightarrow 0$ as $N \rightarrow \infty$ (since $f \in L^{2}\left(\Omega^{d}\right)$ ) and

$$
\lim _{N \rightarrow \infty}\left\|\sup _{0 \leq t \leq \delta}\left|\Phi_{t} f-\Phi_{t}^{N} f\right|\right\|_{L^{2}\left(B_{1}\right)}=0
$$

by assumption (43). Thus we arrive to

$$
\left|\left\{x \in B_{1}: \limsup _{t \rightarrow 0}\left|\Phi_{t} f-f\right|>\lambda\right\}\right|=0
$$

and the statement follows taking the union over $\lambda>0$ and covering $\Omega^{d}$ with a countable collection of balls $B_{1}$.

We combine the following lemma with the embedding contained in Lemma 2.2 to verify the maximal estimate hypothesis of Proposition 3.3 in concrete situations.

Lemma 3.4. Let $p \geq 3$ and $s>\max \left(0, \frac{d}{2}-\frac{2}{p-1}\right)$. Then

$$
\|\mathcal{N}(u)-\mathcal{N}(v)\|_{X^{s,-\frac{1}{2}++}} \lesssim\left(\|u\|_{X^{s, \frac{1}{2}+}}^{p-1}+\|v\|_{X^{s, \frac{1}{2}+}}^{p-1}\right)\|u-v\|_{X^{s, \frac{1}{2}+}}
$$

We postpone the proof of Lemma 3.4 to the end of the section.

We denote $R_{0}=\|f\|_{H^{s}\left(\Omega^{d}\right)}$. Recall that $\eta$ is a smooth cut-off of $[0,1]$. Taking $\delta=\delta\left(R_{0}\right)<1$ sufficiently small and combining (27), (28), (29) and Lemma 3.4 one can show that the map

$$
\Gamma(u(x, t))=\eta(t) e^{i t \Delta} \mathrm{P}_{\leq N} f(x)-i \eta(t) \int_{0}^{t} e^{i\left(t-t^{\prime}\right) \Delta} \mathrm{P}_{\leq N} \mathcal{N}\left(u\left(x, t^{\prime}\right)\right) d t^{\prime}
$$

is a contraction on the ball $\left\{u:\|u\|_{X_{\delta}^{s, \frac{1}{2}+}} \leq 2 R_{0}\right\}$, for all $N \in 2^{\mathbb{N}} \cup\{\infty\}$. This is a standard argument, so we omit the proof (see for instance [22, Section 3.5.1]).

\footnotetext{
${ }^{5}$ Hereafter we remove the $x$ variable in the argument of decompositions like (44) to simplify the notation.
} 
Moreover, a similar computation is part of the proof of Theorem 1.1. However, we stress that the value of $\delta$ is uniform in $N \in 2^{\mathbb{N}} \cup\{\infty\}$. In particular we have

$$
\left\|\Phi_{t}^{N} f\right\|_{X_{\delta}^{s, \frac{1}{2}+}} \leq 2 R_{0}, \quad \text { for all } N \in 2^{\mathbb{N}} \cup\{\infty\} .
$$

We are now ready to prove Theorem 1.1.

Proof of Theorem 1.1. We first prove the a.e. convergence statement (4). By Lemma 2.2 we have

$$
\left\|\sup _{0 \leq t \leq \delta}\left|\Phi_{t} f(x)-\Phi_{t}^{N} f(x)\right|\right\|_{L_{x}^{2}\left(B_{1}\right)} \lesssim\left\|\Phi_{t} f-\Phi_{t}^{N} f\right\|_{X_{\delta}^{s, \frac{1}{2}+}} \cdot
$$

Thus using Proposition 3.3 it suffices to show that the right hand side goes to zero as $N \rightarrow \infty$. For $t \in[0, \delta]$ we have (see (46))

$$
\begin{aligned}
& \Phi_{t} f(x)-\Phi_{t}^{N} f(x) \\
& =\eta(t) e^{i t \Delta} \mathrm{P}_{>N} f(x)-i \eta(t) \int_{0}^{t} e^{i\left(t-t^{\prime}\right) \Delta}\left(\mathcal{N}\left(\Phi_{t^{\prime}} f(x)\right)-\mathrm{P}_{\leq N} \mathcal{N}\left(\Phi_{t^{\prime}}^{N} f(x)\right)\right) d t^{\prime} .
\end{aligned}
$$

Then using (27) and (28) we have

$$
\left\|\Phi_{t} f-\Phi_{t}^{N} f\right\|_{X_{\delta}^{s, \frac{1}{2}+}} \lesssim\left\|\mathrm{P}_{>N} f\right\|_{H^{s}\left(\Omega^{d}\right)}+\left\|\mathcal{N}\left(\Phi_{t} f\right)-\mathrm{P}_{\leq N} \mathcal{N}\left(\Phi_{t}^{N} f\right)\right\|_{X_{\delta}^{s,-\frac{1}{2}+}} .
$$

To handle the nonlinear contribution we further decompose

$$
\mathcal{N}\left(\Phi_{t} f\right)-\mathrm{P}_{\leq N} \mathcal{N}\left(\Phi_{t}^{N} f\right)=\mathrm{P}_{\leq N}\left(\mathcal{N}\left(\Phi_{t} f\right)-\mathcal{N}\left(\Phi_{t}^{N} f\right)\right)+\mathrm{P}_{>N} \mathcal{N}\left(\Phi_{t} f\right)
$$

so that

$$
\begin{array}{r}
\left\|\Phi_{t} f-\Phi_{t}^{N} f\right\|_{X_{\delta}^{s, \frac{1}{2}+}} \lesssim\left\|\mathrm{P}_{>N} f\right\|_{H^{s}\left(\Omega^{d}\right)}+\left\|\mathrm{P}_{>N} \mathcal{N}\left(\Phi_{t} f\right)\right\|_{X_{\delta}^{s,-\frac{1}{2}+}} \\
+\left\|\mathrm{P}_{\leq N}\left(\mathcal{N}\left(\Phi_{t} f\right)-\mathcal{N}\left(\Phi_{t}^{N} f\right)\right)\right\|_{X_{\delta}^{s,-\frac{1}{2}+}}
\end{array}
$$

Then by (29), Lemma 3.4, and (47), we get

$$
\left\|\mathrm{P}_{\leq N}\left(\mathcal{N}\left(\Phi_{t} f\right)-\mathcal{N}\left(\Phi_{t}^{N} f\right)\right)\right\|_{X_{\delta}^{s,-\frac{1}{2}+}} \lesssim \delta^{0+} R_{0}^{p-1}\left\|\Phi_{t} f-\Phi_{t}^{N} f\right\|_{X_{\delta}^{s, \frac{1}{2}+}}
$$

where we recall $R_{0}=\|f\|_{H^{s}\left(\Omega^{d}\right)}$. Plugging (50) into (49), taking $\delta=\delta\left(R_{0}\right)$ small enough and absorbing

$$
\delta^{0+} R_{0}^{p-1}\left\|\Phi_{t} f-\Phi_{t}^{N} f\right\|_{X_{\delta}^{s, \frac{1}{2}+}} \leq \frac{1}{2}\left\|\Phi_{t} f-\Phi_{t}^{N} f\right\|_{X_{\delta}^{s, \frac{1}{2}+}}
$$

into the left hand side, we arrive to

$$
\left\|\Phi_{t} f-\Phi_{t}^{N} f\right\|_{X_{\delta}^{s, \frac{1}{2}+}} \lesssim\left\|\mathrm{P}_{>N} f\right\|_{H^{s}\left(\Omega^{d}\right)}+\left\|\mathrm{P}_{>N} \mathcal{N}\left(\Phi_{t} f\right)\right\|_{X_{\delta}^{s,-\frac{1}{2}+}}
$$

The right hand side of (51) goes to zero as $N \rightarrow \infty$ since $f \in H^{s}\left(\Omega^{d}\right)$ and $\mathcal{N}\left(\Phi_{t} f\right) \in$ $X_{\delta}^{s,-\frac{1}{2}+}$; in fact applying Lemma 3.4 with $v=0$ and recalling (47) we have

$$
\left\|\mathcal{N}\left(\Phi_{t} f\right)\right\|_{X_{\delta}^{s,-\frac{1}{2}+}} \lesssim\left\|\Phi_{t} f\right\|_{X_{\delta}^{s, \frac{1}{2}+}}^{p} \lesssim R_{0}^{p}
$$

This concludes the proof of (4).

To prove (5) it is enough to show that if $d=1,2$ and $s>d / 6$ then

$$
\left\|\int_{0}^{t} e^{i\left(t-t^{\prime}\right) \Delta}\left|\Phi_{t^{\prime}} f\right|^{2} \Phi_{t^{\prime}} f d t^{\prime}\right\|_{X_{\delta}^{\frac{d}{2}+, \frac{1}{2}+}} \lesssim\left\|\Phi_{t} f\right\|_{X_{\delta}^{s, \frac{1}{2}++}}^{3} \lesssim R_{0}^{3} .
$$


Indeed then we would have $\Phi_{t} f-e^{i t \Delta} f \in X_{\delta}^{\frac{d}{2}+, \frac{1}{2}+}$ and we can use $X_{\delta}^{\frac{d}{2}+, \frac{1}{2}+} \hookrightarrow$ $C_{t}\left([0, \delta] ; H^{\frac{d}{2}+}\left(\Omega^{d}\right)\right)$ and $H^{\frac{d}{2}+}\left(\Omega^{d}\right) \hookrightarrow C_{x}\left(\Omega^{d}\right)$ (Sobolev embedding) to get (5). On the other hand (52) follows by (28), Corollary 2.6 and (47), so we are done.

We conclude this section with the proof of Lemma 3.4 and the statement of a similar one - an analog for functions with frequencies restricted to dyadic annuli. These kind of results are now very well understood; however we report the proof for the sake of completeness.

Proof of Lemma 3.4. We consider the case $\Omega=\mathbb{T}$. The proof in the case $\Omega=\mathbb{R}$ requires some modification. It is in fact easier, since there is no loss in the endpoint case $p=2\left(\frac{d+2}{s}\right)$ of the Strichartz estimates (19). We abbreviate everywhere in the proof

$$
L_{x, t}^{q}\left(\mathbb{T}^{d+1}\right) \quad \text { to } \quad L_{x, t}^{q} .
$$

Recalling $\mathcal{N}(z):=|z|^{p-1} z$ and using the Fundamental Theorem of Calculus we can represent

$$
\begin{aligned}
& \mathcal{N}(u)-\mathcal{N}(v)=\int_{0}^{1} \frac{d}{d \rho}(\mathcal{N}(v+\rho(u-v))) d \rho \\
& =(u-v) \int_{0}^{1}\left(\partial_{z} \mathcal{N}\right)(v+\rho(u-v)) d \rho+(\overline{u-v}) \int_{0}^{1}\left(\partial_{\bar{z}} \mathcal{N}\right)(v+\rho(u-v)) d \rho \\
& =:(u-v) \zeta_{1}(u, v)+(\overline{u-v}) \zeta_{2}(u, v) .
\end{aligned}
$$

Notice that $\partial_{z} \mathcal{N}$ and $\partial_{\bar{z}} \mathcal{N}$ are continuous functions since $p \geq 3$. For simplicity we only show that the $X^{s,-\frac{1}{2}++}$ norm of $(u-v) \zeta_{1}(u, v)$ is bounded by the right hand side of (45). The proof that the same holds for $(\overline{u-v}) \zeta_{2}(u, v)$ is identical. Let decompose dyadically

$$
\left\|(u-v) \zeta_{1}(u, v)\right\|_{X^{s,-\frac{1}{2}++}}^{2}=\sum_{N} N^{2 s}\left\|\mathrm{P}_{N}\left((u-v) \zeta_{1}(u, v)\right)\right\|_{X^{0,-\frac{1}{2}++}}^{2}
$$

and estimate

$$
\begin{aligned}
N^{s}\left\|\mathrm{P}_{N}\left((u-v) \zeta_{1}(u, v)\right)\right\|_{X^{0,-\frac{1}{2}++}} & \lesssim N^{s}\left\|\mathrm{P}_{N}\left((u-v) \mathrm{P}_{\ll N} \zeta_{1}(u, v)\right)\right\|_{X^{0,-\frac{1}{2}++}} \\
& +\sum_{N_{1} \gtrsim N} N^{s}\left\|\mathrm{P}_{N}\left((u-v) \mathrm{P}_{N_{1}} \zeta_{1}(u, v)\right)\right\|_{X^{0,-\frac{1}{2}++}}
\end{aligned}
$$

We first focus on the second term on the right hand side of (55). This one is the easiest to bound, since the restriction on frequencies $N_{1} \gtrsim N$ gives a gain once we estimate the norm of $\mathrm{P}_{N_{1}} \zeta_{1}(u, v)$. Using (24), Hölder's inequality, and (23) we get

$$
\begin{aligned}
& \sum_{N_{1} \gtrsim N} N^{s}\left\|\mathrm{P}_{N}\left((u-v) \mathrm{P}_{N_{1}} \zeta_{1}(u, v)\right)\right\|_{X^{0,-\frac{1}{2}++}} \\
& \lesssim \sum_{N_{1} \gtrsim N} N^{s+}\left\|(u-v) \mathrm{P}_{N_{1}} \zeta_{1}(u, v)\right\|_{L_{x, t}^{2\left(\frac{d+2}{d+4}\right)+}} \\
& \lesssim \sum_{N_{1} \gtrsim N} N^{s+}\|u-v\|_{L_{x, t}^{2\left(\frac{d+2}{d}\right)}}\left\|\mathrm{P}_{N_{1}} \zeta_{1}(u, v)\right\|_{L_{x, t}^{\frac{d+2}{2}+}} \\
& \lesssim \sum_{N_{1} \gtrsim N} N^{0+}\|u-v\|_{X^{s, \frac{1}{2}+}}\left\|\mathrm{P}_{N_{1}} \zeta_{1}(u, v)\right\|_{L_{x, t}^{\frac{d+2}{2}+}}
\end{aligned}
$$


Recalling the definition of $\zeta_{1}(u, v)$ and using Minkowski's inequality and $L^{p}$ estimates for nonlinear operators of power type (see for instance [17, Proposition 2.3]) we have

$$
\begin{aligned}
& \left\|\mathrm{P}_{N_{1}} \zeta_{1}(u, v)\right\|_{L_{x, t}^{\frac{d+2}{2}+}} \lesssim N_{1}^{0+}\left(\begin{array}{cc}
\|u\|^{p-2} \\
L_{x, t}^{2\left(\frac{d+2}{d-2 s}\right)-}
\end{array}+\|v\|_{L_{x, t}^{2\left(\frac{d+2}{d-2 s}\right)-}}^{p-2}\right) \\
& \times \sum_{N_{2}} \min \left(1, \frac{N_{2}}{N_{1}}\right)\left(\left\|\mathrm{P}_{N_{2}} u\right\|_{L_{x, t}^{\frac{2(d+2)(d-2 s)}{4-(p-2)}}+}+\left\|\mathrm{P}_{N_{2}} v\right\|_{L_{x, t}^{\frac{2-(p-2)(d-2 s)}{4}}+}\right) .
\end{aligned}
$$

Notice that (23) gives

$$
\left\|\mathrm{P}_{N_{2}} F(x, t)\right\|_{L_{x, t}^{\frac{2(d-2)(d-2)}{4-(p-2)}}+} \lesssim N_{2}^{0---}\left\|\mathrm{P}_{N_{2}} F(x, t)\right\|_{X^{s, \frac{1}{2}+}} .
$$

Indeed, if $\frac{2(d+2)}{4-(p-2)(d-2 s)} \geq \frac{2(d+2)}{d}$ we can use (23) and we get a factor $N_{2}^{\frac{(p-1)(d-2 s)-4}{2}+}$. Since $\frac{(p-1)(d-2 s)-4}{2}+<0$ for $s>\frac{d}{2}-\frac{2}{p-1}$ we get (58). If $\frac{2(d+2)}{4-(p-2)(d-2 s)} \leq \frac{2(d+2)}{d}$ we can bound the $L_{x, t}^{\frac{2(d+2)}{4-(p-2)(d-2 s)}}$ norm with the $L_{x, t}^{\frac{2(d+2)}{d}}$ norm and use (23), to get a factor $N_{2}^{-s+}$. Again, since $s>0$ we get (58). Using (58) and (25) the estimate (57) becomes

$$
\begin{aligned}
\| \mathrm{P}_{N_{1}} \zeta_{1}(u, v) & \|_{L_{x, t}^{\frac{d+2}{2}+}} \lesssim N_{1}^{0+}\left(\|u\|_{X^{s, \frac{1}{2}+}}^{p-2}+\|v\|_{X^{s, \frac{1}{2}+}}^{p-2}\right) \\
& \times \sum_{N_{2}} \min \left(1, \frac{N_{2}}{N_{1}}\right) N_{2}^{0--}\left(\left\|\mathrm{P}_{N_{2}} u\right\|_{X^{s, \frac{1}{2}+}}+\left\|\mathrm{P}_{N_{2}} v\right\|_{X^{s, \frac{1}{2}+}}\right) \\
& \lesssim N_{1}^{0--}\left(\|u\|_{X^{s, \frac{1}{2}+}}^{p-1}+\|v\|_{X^{s, \frac{1}{2}+}}^{p-1}\right)
\end{aligned}
$$

Plugging (59) into (56) we obtain (recall $N_{1} \gtrsim N$ )

$$
\begin{aligned}
& \sum_{N_{1} \gtrsim N} N^{s}\left\|\mathrm{P}_{N}\left((u-v) \mathrm{P}_{N_{1}} \zeta_{1}(u, v)\right)\right\|_{X^{0,-\frac{1}{2}++}} \\
& \lesssim \sum_{N_{1} \gtrsim N} N_{1}^{0-}\|u-v\|_{X^{s, \frac{1}{2}+}}\left(\|u\|_{X^{s, \frac{1}{2}+}}^{p-1}+\|v\|_{X^{s, \frac{1}{2}+}}^{p-1}\right) \\
& \lesssim N^{0-}\|u-v\|_{X^{s, \frac{1}{2}+}}\left(\|u\|_{X^{s, \frac{1}{2}+}}^{p-1}+\|v\|_{X^{s, \frac{1}{2}+}}^{p-1}\right) .
\end{aligned}
$$

Summing the square of this inequality over $N$, we have handled the contribution of the second term on the right hand side of (55). To handle the first term we note that

(60)

$N^{s}\left\|\mathrm{P}_{N}\left((u-v) \mathrm{P}_{\ll N} \zeta_{1}(u, v)\right)\right\|_{X^{0,-\frac{1}{2}++}} \lesssim N^{s}\left\|\left(\mathrm{P}_{\sim N}(u-v)\right) \mathrm{P}_{\ll N} \zeta_{1}(u, v)\right\|_{X^{0,-\frac{1}{2}++}}$ and decompose

$$
\begin{aligned}
\left(\mathrm{P}_{\sim N}(u-v)\right) \mathrm{P}_{\ll N} \zeta_{1}(u, v) & =\sum_{N_{1} \ll N} \sum_{Q_{N, N_{1}}}\left(\mathrm{P}_{Q_{N, N_{1}}}(u-v)\right) \mathrm{P}_{N_{1}} \zeta_{1}(u, v) \\
& =\sum_{N_{1} \ll N} \sum_{Q_{N, N_{1}}} \mathrm{P}_{100 Q_{N, N_{1}}}\left(\left(\mathrm{P}_{Q_{N, N_{1}}}(u-v)\right) \mathrm{P}_{N_{1}} \zeta_{1}(u, v)\right),
\end{aligned}
$$


where $Q_{N, N_{1}}$ is a partition of the annulus of size $N$ into cubes of side $N_{1}$ (this is possible since $\left.N_{1}<N\right)$. In the second identity we used that the support of $\left(\mathrm{P}_{Q_{N, N_{1}}} F\right) \mathrm{P}_{N_{1}} G$ is contained in $100 Q_{N, N_{1}}$. Since for different $N, N_{1}$ the projections $\mathrm{P}_{Q_{N, N_{1}}}$ are (almost) orthogonal, squaring (61) we get

$$
\begin{aligned}
& N^{2 s}\left\|\mathrm{P}_{N}\left((u-v) \mathrm{P}_{\ll N} \zeta_{1}(u, v)\right)\right\|_{X^{0,-\frac{1}{2}++}}^{2} \\
& \quad=N^{2 s} \sum_{N_{1} \ll N} \sum_{Q_{N, N_{1}}}\left\|\mathrm{P}_{100 Q_{N, N_{1}}}\left(\left(\mathrm{P}_{Q_{N, N_{1}}}(u-v)\right) \mathrm{P}_{N_{1}} \zeta_{1}(u, v)\right)\right\|_{X^{0,-\frac{1}{2}++}}^{2} .
\end{aligned}
$$

Proceeding exactly as before we get

$$
\begin{aligned}
\| \mathrm{P}_{100 Q_{N, N_{1}}} & \left(\left(\mathrm{P}_{Q_{N, N_{1}}}(u-v)\right) \mathrm{P}_{N_{1}} \zeta_{1}(u, v)\right) \|_{X^{0,-\frac{1}{2}++}} \\
& \lesssim N_{1}^{0-}\left\|\mathrm{P}_{Q_{N, N_{1}}}(u-v)\right\|_{X^{0, \frac{1}{2}+}}\left(\|u\|_{X^{s, \frac{1}{2}+}}^{p-1}+\|v\|_{X^{s, \frac{1}{2}+}}^{p-1}\right)
\end{aligned}
$$

notice that since the side of $Q_{N, N_{1}}$ is $N_{1}$ we had only powers of $N_{1}$ in this computation. Thus

$$
\begin{aligned}
& N^{2 s}\left\|\mathrm{P}_{N}\left((u-v) \mathrm{P}_{\ll N} \zeta_{1}(u, v)\right)\right\|_{X^{0,-\frac{1}{2}++}}^{2} \lesssim \\
& \quad\left(\|u\|_{X^{s, \frac{1}{2}+}}^{p-1}+\|v\|_{X^{s, \frac{1}{2}+}}^{p-1}\right)^{2} N^{2 s} \sum_{N_{1} \ll N} \sum_{Q_{N, N_{1}}} N_{1}^{0-}\left\|\mathrm{P}_{Q_{N, N_{1}}}(u-v)\right\|_{X^{0, \frac{1}{2}+}}^{2} .
\end{aligned}
$$

Summing the square of (65) over $Q_{N, N_{1}}$ (recall that these cubes are a partition of the annulus of size $N$ ) and later over $N_{1}$, we obtain (after taking the square root)

$N^{s}\left\|\mathrm{P}_{N}\left((u-v) \mathrm{P}_{\ll N} \zeta_{1}(u, v)\right)\right\|_{X^{0,-\frac{1}{2}++}} \lesssim\left(\|u\|_{X^{s, \frac{1}{2}+}}^{p-1}+\|v\|_{X^{s, \frac{1}{2}+}}^{p-1}\right)\left\|\mathrm{P}_{N}(u-v)\right\|_{X^{s, \frac{1}{2}+}}$,

which gives the correct control also on the first term on the right hand side of (55). This concludes the proof.

Later we will also need the following Lemma, whose proof is a straightforward adaptation of the previous argument.

Lemma 3.5. Let $d=2$ and $s>0$. Let $M_{1} \geq M_{2} \geq M_{3}$ be dyadic scales. Then

$$
\begin{aligned}
\left\|\left(\mathrm{P}_{M_{1}} F\right)\left(\mathrm{P}_{M_{2}} G\right)\left(\mathrm{P}_{M_{3}} H\right)\right\|_{X^{s,-\frac{1}{2}++}} & \\
& \lesssim\left\|\mathrm{P}_{M_{1}} F\right\|_{X^{s, \frac{1}{2}+}}\left\|\mathrm{P}_{M_{2}} G\right\|_{X^{0+, \frac{1}{2}+}}\left\|\mathrm{P}_{M_{3}} H\right\|_{X^{0, \frac{1}{2}+}}
\end{aligned}
$$

\section{Probabilistic Results}

4.1. The Linear Schrödinger Equation on $\mathbb{T}^{d}$ with Random Data. Here we prove almost surely uniform convergence of the randomized Schrödinger flow to the initial datum, at the $H^{0+}$ level. More precisely, we show that $e^{i t \Delta} f^{\omega} \rightarrow f^{\omega}$ as $t \rightarrow 0$ uniformly over $x \in \mathbb{T}^{d}$ and $\mathbb{P}$-almost surely for data $f^{\omega}$ defined as

$$
f^{\omega}(x)=\sum_{n \in \mathbb{Z}^{d}} \frac{g_{n}^{\omega}}{\langle n\rangle^{\frac{d}{2}+\alpha}} e^{i n \cdot x}, \quad x \in \mathbb{T}^{d},
$$

where $\alpha>0$ and each $g_{n}^{\omega}$ is complex and independently drawn from a standard normal distribution. In fact, the argument we present works for independent $g_{n}^{\omega}$ 
drawn from any distribution with sufficiently strong decay properties. We present the standard normal case for definiteness. Fix $t \in \mathbb{R}$. We have that $\mathbb{P}$-almost surely

$$
e^{i t \Delta} f^{\omega} \in \bigcap_{s<\alpha} H^{s}\left(\mathbb{T}^{d}\right)
$$

This is an immediate consequence of (75) below, taking the union over $\varepsilon>0$. Moreover, for all $t \in \mathbb{R}$ the $e^{i t \Delta} f^{\omega}$ are $\mathbb{P}$-almost surely continuous functions ${ }^{6}$ of the $x$ variable. This is a consequence of the higher integrability property (72) below, from which one can easily deduce uniform convergence as $N \rightarrow \infty$ of the sequence $P_{\leq N} f^{\omega}$, with probability larger than $1-\varepsilon$. So the limit $f^{\omega}$ is continuous with the same probability, and the almost sure continuity follows taking the union over $\varepsilon>0$.

Now we prove the first part of Theorem 1.3, namely

Proposition 4.1. Let $\alpha>0$. For $\mathbb{P}$-almost every $f^{\omega}$ of the form (8) we have that

$$
e^{i t \Delta} f^{\omega}(x) \rightarrow f^{\omega}(x) \quad \text { as } t \rightarrow 0
$$

for every $x \in \mathbb{T}^{d}$ and uniformly.

This proposition proves the first part of Theorem 1.3. Its proof appears at the end of this section after we establish few lemmata.

We start recalling the following well-known concentration bound:

Lemma 4.2 ([9, Lemma 3.1]). There exists a constant $C$ such that

$$
\left\|\sum_{n \in \mathbb{Z}^{d}} g_{n}^{\omega} a_{n}\right\|_{L_{\omega}^{r}} \leq C r^{\frac{1}{2}}\left\|a_{n}\right\|_{\ell_{n}^{2}\left(\mathbb{Z}^{d}\right)}
$$

for all $r \geq 2$ and $\left\{a_{n}\right\} \in \ell^{2}\left(\mathbb{Z}^{d}\right)$.

Using (68) with $a_{n}=e^{i n \cdot x-i|n|^{2} t}\langle n\rangle^{-\frac{d}{2}-\alpha}$ we obtain for $r \geq 2$ that for $f^{\omega}$ an in $(67)$

$$
\left\|\mathrm{P}_{N} e^{i t \Delta} f^{\omega}\right\|_{L_{\omega}^{r}} \leq C r^{\frac{1}{2}} N^{-\alpha},
$$

with a constant uniform in $t \in \mathbb{R}$. From this, we also have improved $L_{x}^{p}$ estimates for randomized data.

Lemma 4.3. Let $p \in[2, \infty)$. Assume $f^{\omega}$ is as in (67). There exists constants $C$ and $c$, independent of $t \in \mathbb{R}$, such that

$$
\mathbb{P}\left(\left\|\mathrm{P}_{N} e^{i t \Delta} f^{\omega}\right\|_{L_{x}^{p}\left(\mathbb{T}^{d}\right)}>\lambda\right) \leq C e^{-c N^{2 \alpha} \lambda^{2}} .
$$

In particular, for any $\varepsilon>0$ sufficiently small, we have

$$
\left\|\mathrm{P}_{N} e^{i t \Delta} f^{\omega}\right\|_{L_{x}^{p}\left(\mathbb{T}^{d}\right)} \lesssim N^{-\alpha}(-\ln \varepsilon)^{1 / 2}, \quad N \in 2^{\mathbb{Z}} \cup\{\infty\}
$$

with probability at least $1-\varepsilon$. Thus

$$
\left\|\mathrm{P}_{N} e^{i t \Delta} f^{\omega}\right\|_{L_{x}^{\infty}\left(\mathbb{T}^{d}\right)} \lesssim N^{-\alpha+}(-\ln \varepsilon)^{1 / 2}, \quad N \in 2^{\mathbb{Z}} \cup\{\infty\},
$$

with probability at least $1-\varepsilon$.

\footnotetext{
${ }^{6}$ In fact they belong to $\bigcap_{s<\alpha} C^{s}\left(\mathbb{T}^{d}\right) \mathbb{P}$-almost surely, but we will never need this stronger information.
} 
Proof. We prove (71), then (72) follows by Bernstein inequality. By Minkowski's inequality and Lemma 4.2 above, we have for any $r \geq p \geq 2$

$$
\left(\int\left\|\mathrm{P}_{N} e^{i t \Delta} f^{\omega}\right\|_{L_{x}^{p}\left(\mathbb{T}^{d}\right)}^{r} d \mathbb{P}(\omega)\right)^{\frac{1}{r}} \leq\|\| \mathrm{P}_{N} e^{i t \Delta} f^{\omega}\left\|_{L_{\omega}^{r}}\right\|_{L_{x}^{p}\left(\mathbb{T}^{d}\right)} \leq C N^{-\alpha} r^{\frac{1}{2}} .
$$

which is enough to conclude that $\left\|\mathrm{P}_{N} e^{i t \Delta} f^{\omega}\right\|_{L_{x}^{p}\left(\mathbb{T}^{d}\right)}$ is a sub-Gaussian random variable satisfying the tail bound (70).

Proceeding as in the proof of Lemma 4.3 we also obtain improved Strichartz estimates for randomized data.

Lemma 4.4. Let $p \in[2, \infty)$. Assume $f^{\omega}$ is as in (67). Then we have, for some constants $C$ and $c$, independent of $t \in \mathbb{R}$ the bound

$$
\mathbb{P}\left(\left\|e^{i t \Delta} \mathrm{P}_{N} f^{\omega}\right\|_{L_{x, t}^{p}\left(\mathbb{T}^{d+1}\right)}>\lambda\right) \leq C e^{-c N^{2 \alpha} \lambda^{2}} .
$$

In particular, for any $\varepsilon>0$ sufficiently small, we have

$$
\left\|e^{i t \Delta} \mathrm{P}_{N} f^{\omega}\right\|_{L_{x, t}^{p}\left(\mathbb{T}^{d+1}\right)} \lesssim N^{-\alpha}(-\ln \varepsilon)^{1 / 2}, \quad N \in 2^{\mathbb{Z}} \cup\{\infty\},
$$

with probability at least $1-\varepsilon$. Thus

$$
\left\|e^{i t \Delta} \mathrm{P}_{N} f^{\omega}\right\|_{L_{x, t}^{\infty}\left(\mathbb{T}^{d+1}\right)} \lesssim N^{-\alpha+}(-\ln \varepsilon)^{1 / 2}, \quad N \in 2^{\mathbb{Z}} \cup\{\infty\},
$$

with probability at least $1-\varepsilon$.

Fix $t \in \mathbb{R}$. Later we will also need the following bound (with high probability) for the $H^{s}$ norm of $e^{i t \Delta} f^{\omega}$ with $s<\alpha$. This is a well know fact that we recall applying again (68) with $a_{n}=e^{i n \cdot x-|n|^{2} t}\langle n\rangle^{-\frac{d}{2}-\alpha+s}$, so that we get for $r \geq 2$

$$
\left\|\mathrm{P}_{N}\langle D\rangle^{s} e^{i t \Delta} f^{\omega}\right\|_{L_{\omega}^{r}} \leq C r^{\frac{1}{2}} N^{s-\alpha}, \quad s<\alpha .
$$

Here $\langle D\rangle$ denotes the Fourier multiplier operator $\langle n\rangle$. Proceeding as in the proof of Lemma 4.3 we also obtain

$$
\mathbb{P}\left(\left\|\langle D\rangle^{s} \mathrm{P}_{N} e^{i t \Delta} f^{\omega}\right\|_{L_{x}^{2}\left(\mathbb{T}^{d}\right)}>\lambda\right) \leq C e^{-c N^{2(\alpha-s)} \lambda^{2}}, \quad s<\alpha
$$

and in particular, for any $\varepsilon>0$ sufficiently small

$$
\left\|e^{i t \Delta} f^{\omega}\right\|_{H_{x}^{s}\left(\mathbb{T}^{d}\right)} \lesssim(-\ln \varepsilon)^{1 / 2} \quad s<\alpha, \quad t \in \mathbb{R},
$$

with probability at least $1-\varepsilon$. Again the constant is uniform on $t \in \mathbb{R}$.

We thanks Chenjie Fan for sharing with use the argument we used in the next proof, and that strengthens our original a.e. convergence to a uniform one.

Proof of Proposition 4.1. Let us decompose

$$
\left|e^{i t \Delta} f^{\omega}-f^{\omega}\right| \leq\left|e^{i t \Delta} \mathrm{P}_{>N} f^{\omega}\right|+\left|e^{i t \Delta} \mathrm{P}_{\leq N} f^{\omega}-\mathrm{P}_{\leq N} f^{\omega}\right|+\left|\mathrm{P}_{>N} f^{\omega}\right| .
$$

We fix $\lambda>0$ and $\varepsilon>0$ sufficiently small. Using (74) we see that

$$
\left\|e^{i t \Delta} \mathrm{P}_{>N} f^{\omega}\right\|_{L_{x, t}^{\infty}\left(\mathbb{T}^{d+1}\right)}+\left\|\mathrm{P}_{>N} f^{\omega}\right\|_{L_{x}^{\infty}\left(\mathbb{T}^{d}\right)}<\lambda / 2
$$

holds for all $N$ sufficiently large, depending on $\lambda$, $\varepsilon$, with probability larger than $1-\varepsilon$. Let us fix such $N^{*}=N^{*}(\lambda, \varepsilon)$. We also fix $s^{*}>\frac{d}{2}$. Since

$$
e^{i t \Delta} \mathrm{P}_{\leq N^{*}} f^{\omega}-\mathrm{P}_{\leq N *} f^{\omega}=\sum_{|n| \leq N^{*}}\left(e^{-i t|n|^{2}}-1\right) e^{i n \cdot x} \widehat{f^{\omega}}(n),
$$


using Cauchy-Schwartz, the summability of $\langle n\rangle^{-2 s^{*}}$ and (75) with $s=0, t=0$ (in the last inequality) we get

$$
\begin{aligned}
\left\|e^{i t \Delta} \mathrm{P}_{\leq N^{*}} f^{\omega}-\mathrm{P}_{\leq N *} f^{\omega}\right\|_{L_{\mathbb{T}^{d}}^{\infty}} & \lesssim \sup _{|n| \leq N^{*}}\left|e^{-i t|n|^{2}}-1\right|\left(\sum_{|n| \leq N^{*}}\langle n\rangle^{2 s^{*}}\left|\widehat{f^{\omega}}(n)\right|^{2}\right)^{1 / 2} \\
& \lesssim|t|\left(N^{*}\right)^{s^{*}+2}\left\|f^{\omega}\right\|_{L^{2}} \lesssim|t|\left(N^{*}\right)^{s^{*}+2}(-\ln \varepsilon)^{1 / 2}
\end{aligned}
$$

with probability larger than $1-\varepsilon$. Thus we can find $t^{*}$ sufficiently small, depending only on $N^{*}$ and $\varepsilon$, such that for all $t \in\left(0, t^{*}\right)$ we have

$$
\left\|e^{i t \Delta} \mathrm{P}_{\leq N^{*}} f^{\omega}-\mathrm{P}_{\leq N^{*}} f^{\omega}\right\|_{L_{x}^{\infty}\left(\mathbb{T}^{d}\right)}<\lambda / 2
$$

with probability larger than $1-\varepsilon$. Plugging (77), (79) into (76) we see the following. Given $\lambda>0$, we have found $t^{*}=t^{*}(\lambda, \varepsilon)$ such that

$$
\left\|e^{i t \Delta} f^{\omega}-f^{\omega}\right\|_{L_{x}^{\infty}\left(\mathbb{T}^{d}\right)}<\lambda, \quad \text { for all } t \in\left(0, t^{*}\right),
$$

with probability larger than $1-\varepsilon$. Namely for all $\varepsilon>0$ we have uniform (in $x$ ) convergence $e^{i t \Delta} f^{\omega} \rightarrow f^{\omega}$ as $t \rightarrow 0$ for all $\omega \in A_{\delta}$ with $\mathbb{P}\left(A_{\delta}\right)>1-2 \varepsilon$. This means that the uniform convergence fails only if $\omega$ belongs to any of the complementary sets $A_{\varepsilon}^{C}$, thus in particular for $\omega \in \bigcap_{k \in \mathbb{N}} A_{1 / k}$. Since Since $\mathbb{P}\left(\bigcap_{k \in \mathbb{N}} A_{1 / k}\right)=$ $\lim _{k \rightarrow 0} \mathbb{P}\left(A_{1 / k}\right)=0$ the statement follows.

\subsection{The Linear Schrödinger Equation on $\mathbb{R}^{d}$ with Random Data.}

For the linear Schrödinger equation on $\mathbb{R}^{d}$, randomization arguments similar to those in Section 4.1 can be applied. Given $s>0$ and $f \in H^{s}\left(\mathbb{R}^{d}\right)$, we work with the randomized data $f^{\omega}$ defined in (14). As in the periodic case, this argument works for any independent random variables whose distribution functions decay sufficiently rapidly. We work with the standard normal distribution for the sake of definiteness.

By arguments almost identical to those for the periodic case, we have for any $p \in[2, \infty)$

$$
\left\|P_{N} e^{i t \Delta} f^{\omega}\right\|_{L^{p}\left(\mathbb{R}^{d} \times[0,1]\right)} \lesssim N^{-s}(-\ln \varepsilon)^{1 / 2}\left\|P_{N} f\right\|_{L^{2}\left(\mathbb{R}^{d}\right)}, \quad N \in 2^{\mathbb{Z}} \cup\{\infty\},
$$

for $\omega$ in a set of probability at least $1-\varepsilon$. Thus the Bernstein inequality gives

$$
\left\|P_{N} e^{i t \Delta} f^{\omega}\right\|_{L^{\infty}\left(\mathbb{R}^{d} \times[0,1]\right)} \lesssim N^{-s+}(-\ln \varepsilon)^{1 / 2}\left\|P_{N} f\right\|_{L^{2}\left(\mathbb{R}^{d}\right)}, \quad N \in 2^{\mathbb{Z}} \cup\{\infty\},
$$

for $\omega$ in a set of probability at least $1-\varepsilon$. In particular $e^{i t \Delta} f^{\omega}$ is $\mathbb{P}$-almost surely continuous ${ }^{7}$.

Moreover one has uniform in $t \in \mathbb{R}$ bounds for the $H^{s}$ norm

$$
\left\|e^{i t \Delta} f^{\omega}\right\|_{H^{s}\left(\mathbb{R}^{d}\right)} \lesssim(-\ln \varepsilon)^{1 / 2}\|f\|_{H^{s}\left(\mathbb{R}^{d}\right)},
$$

for $\omega$ in a set of probability at least $1-\varepsilon$. For more general versions of these estimates we refer to [36, Lemmata $2.1 \& 2.3]$.

Using these estimates and proceeding exactly as in the periodic case, we can establish the first part of Theorem 1.5, namely

\footnotetext{
${ }^{7}$ In fact one can show it is $\mathbb{P}$-almost surely in $C_{x}^{s^{\prime}}$ for all $s^{\prime}<s$.
} 
Proposition 4.5. Let $s>0$ and $f \in H^{s}\left(\mathbb{R}^{d}\right)$. For $\mathbb{P}$-almost every $f^{\omega}$ of the form (14) we have

$$
e^{i t \Delta} f^{\omega}(x) \rightarrow f^{\omega}(x) \quad \text { as } t \rightarrow 0
$$

for every $x \in \mathbb{R}^{d}$ and uniformly.

4.3. The Cubic NLS Equation on $\mathbb{T}^{d}(d=1,2)$ with Random Data (Theorem 1.3).

In this section, we consider the cubic Wick-ordered NLS $(9)$ on $\mathbb{T}^{d}(d=1,2)$ as in the work of Bourgain in [5]. Namely, we look at the nonlinearity

$$
\mathcal{N}(u):= \pm u\left(|u|^{2}-2 \mu\right), \quad \mu:=f_{\mathbb{T}^{d}}|u(x, t)|^{2} d x .
$$

We are interested again in randomized initial data, i.e. $f^{\omega}$ is taken to be of the form (67). Recall (see (75)) that such data is $\mathbb{P}$-almost surely in $H^{s}$ for all $s<\alpha$ and

$$
\left\|f^{\omega}\right\|_{H^{s}} \lesssim(-\ln \varepsilon)^{1 / 2}, \quad s<\alpha,
$$

with probability at least $1-\varepsilon$, for all $\varepsilon \in(0,1)$ sufficiently small. Since we work with any $\alpha>0$, we are considering initial data in $H^{0+}$. We approximate equation (9) as in (42), for all $N \in 2^{\mathbb{N}} \cup\{\infty\}$. Recall that $\Phi_{t}^{N} f^{\omega}$ denotes the associated flow, with initial datum

$$
\Phi_{0}^{N} f^{\omega}:=\mathrm{P}_{\leq N} f^{\omega}=\sum_{|n| \leq N} \frac{g_{n}^{\omega}}{\langle n\rangle^{\frac{d}{2}+\alpha}} e^{i n \cdot x} .
$$

We write $\Phi_{t} f^{\omega}=\Phi_{t}^{\infty} f^{\omega}$ for the flow of (9) with datum $f^{\omega}=\mathrm{P}_{\infty} f^{\omega}$.

Proposition 4.6. Let $d=1,2$ and $\alpha>0$. Let $N \in 2^{\mathbb{N}} \cup\{\infty\}$. For all $\sigma \in\left[0, \frac{1}{2}\right)$, the following holds. Assume

$$
u=u(I)+u(I I), \quad u(I)=e^{i t \Delta} \mathrm{P}_{\leq N} f^{\omega}, \quad\|u(I I)\|_{X^{\alpha+\sigma, \frac{1}{2}+}}<1
$$

and the same for $v$. Then

$$
\begin{gathered}
\|\mathcal{N}(u)\|_{X^{\alpha+\sigma,-\frac{1}{2}+}} \lesssim(-\ln \varepsilon)^{3 / 2} \\
\|\mathcal{N}(u)-\mathcal{N}(v)\|_{X^{\alpha+\sigma,-\frac{1}{2}++}} \lesssim(-\ln \varepsilon)\|u-v\|_{X^{\alpha+\sigma, \frac{1}{2}+}}
\end{gathered}
$$

for initial data of the form (67), with probability at least $1-\varepsilon$, for all $\varepsilon \in(0,1)$ sufficiently small. If we take $u$ as in (84) and we instead assume

$$
v=v(I)+u(I I), \quad v(I)=e^{i t \Delta} f^{\omega}, \quad\|u(I I)\|_{X^{\alpha+\sigma, \frac{1}{2}+}}<1,
$$

we have

$$
\|\mathcal{N}(u)-\mathcal{N}(v)\|_{X^{\alpha+\sigma,-\frac{1}{2}++}} \lesssim N^{-\alpha} .
$$

Remark 4.7. Recall that $\alpha$ indicates the regularity of the initial datum. We are denoting by $\sigma$ the amount of smoothing we can prove for the Wick-ordered cubic nonlinearity $\mathcal{N}$. More precisely, since the initial data (67) belongs to $H^{\alpha-}$, we can interpret this statement as saying that, with arbitrarily large probability, $\mathcal{N}$ is $\sigma+$ smoother than $f^{\omega}$. Since $\sigma<\frac{1}{2}$ is permissible, we reach $\frac{1}{2}-$ smoothing for $\mathcal{N}$ and, combining with (28), also for the Duhamel contribution $\Phi_{t}^{N} f^{\omega}-e^{i t \Delta} P_{\leq N} f^{\omega}$. 
We postpone the proof of Proposition 4.6 to the end of the section. Recall that $\eta$ is a smooth cut-off of the unit interval. Let us fix $\alpha>0$. Using (28), (29) and Proposition 4.6 one can show that for all $\delta>0$ sufficiently small the following holds. For all $N \in 2^{\mathbb{N}} \cup\{\infty\}$, the map

$$
\Gamma^{N}(u):=\eta(t) e^{i t \Delta} \mathrm{P}_{\leq N} f^{\omega}-i \eta(t) \int_{0}^{t} e^{i(t-s) \Delta} \mathrm{P}_{\leq N} \mathcal{N}(u(\cdot, s)) d s
$$

is a contraction on the set

$$
\left\{e^{i t \Delta} \mathrm{P}_{\leq N} f^{\omega}+g, \quad\|g\|_{X_{\delta}^{\alpha+\sigma, \frac{1}{2}+}}<1\right\}
$$

equipped with the $X_{\delta}^{\alpha+\sigma, \frac{1}{2}+}$ norm, outside an exceptional set (we call it a $\delta$ exceptional set) of initial data of probability smaller than $e^{-\delta^{-\gamma}}$, with $\gamma>0$ a given small constant. Notice that this holds uniformly over $N \in 2^{\mathbb{N}} \cup\{\infty\}$. Again, this is a standard routine calculation that we omit (see for instance [22, Section 3.5.1]). We only explain how to find the relation between the local existence time $\delta$ and the size of the exceptional set. Given any $\varepsilon \in(0,1)$ sufficiently small, using (28), (29) and Proposition 4.6, we have

$$
\left\|\Gamma^{N}(u)-\eta(t) e^{i t \Delta} \mathrm{P}_{\leq N} f^{\omega}\right\|_{X_{\delta}^{\alpha+\sigma, \frac{1}{2}+}} \lesssim \delta^{0+}(-\ln \varepsilon)^{3 / 2},
$$

for all $f^{\omega}$ outside an exceptional set of probability smaller than $\varepsilon$. Letting $\delta$ such that $\varepsilon=e^{-\delta^{-\gamma}}$ with $\gamma>0$ a fixed small constant, we have $C \delta^{0+}(-\ln \varepsilon)^{3 / 2}<1$ for all $\delta>0$ sufficiently small. Note that the measure $e^{-\delta^{-\gamma}}$ of the $\delta$-exceptional set converges to zero as $\delta \rightarrow 0$. In particular, for $\omega$ outside the $\delta$-exceptional set, the fixed point $\Phi_{t}^{N} f^{\omega}$ of the map (88) belongs to the set (89), namely

$$
\left\|\Phi_{t}^{N} f^{\omega}-e^{i t \Delta} \mathrm{P}_{\leq N} f^{\omega}\right\|_{X_{\delta}^{\alpha+\sigma, \frac{1}{2}+}}<1, \quad N \in 2^{\mathbb{N}} \cup\{\infty\} .
$$

We are now ready to prove Theorem 1.3.

Proof of Theorem 1.3. Notice that (10) is the content of Proposition 4.1. To prove (11), let us assume that we have proved

$$
\lim _{N \rightarrow \infty}\left\|\sup _{0 \leq t \leq \delta}\left|\Phi_{t} f^{\omega}(x)-\Phi_{t}^{N} f^{\omega}(x)\right|\right\|_{L_{x}^{2}\left(\mathbb{T}^{2}\right)}=0
$$

for all $f^{\omega}$ outside a $\delta$-exceptional set $A_{\delta}$. This means that given $f^{\omega}$ we can find, $\mathbb{P}$-almost surely, a $\delta_{\omega}$ such that (91) is satisfied. Indeed, if we could not do so, this would mean that $f^{\omega} \in \bigcap_{\delta>0} A_{\delta}$, and the probability of this event is zero, since $\mathbb{P}\left(A_{\delta}\right) \rightarrow 0$ as $\delta \rightarrow 0$. So, using Proposition 3.3 with $\delta=\delta_{\omega}$, we have $\mathbb{P}$-almost surely

$$
\lim _{t \rightarrow 0} \Phi_{t}^{\omega} f^{\omega}(x)-f^{\omega}(x)=0, \quad \text { for a.e. } x \in \mathbb{T}^{2},
$$

as claimed. It remains to prove (91). We decompose

$$
\left|\Phi_{t} f^{\omega}-\Phi_{t}^{N} f^{\omega}\right| \leq\left|e^{i t \Delta} \mathrm{P}_{>N} f^{\omega}\right|+\left|\Phi_{t} f^{\omega}-e^{i t \Delta} f^{\omega}-\left(\Phi_{t}^{N} f^{\omega}-e^{i t \Delta} \mathrm{P}_{\leq N} f^{\omega}\right)\right|,
$$

Thus, recalling the decay of the high frequency linear term given by (74), it remains to show that

$$
\lim _{N \rightarrow \infty}\left\|\sup _{0 \leq t \leq \delta}\left|\Phi_{t} f^{\omega}-e^{i t \Delta} f^{\omega}-\left(\Phi_{t}^{N} f^{\omega}-e^{i t \Delta} \mathrm{P}_{\leq N} f^{\omega}\right)\right|\right\|_{L^{2}\left(\mathbb{T}^{2}\right)}=0,
$$

for all $f^{\omega}$ outside a $\delta$-exceptional set. 
For any $\alpha>0$, we can choose $\sigma$ sufficiently close to $\frac{1}{2}$ that

$$
s_{\mathbb{T}}<s_{\mathbb{T}^{2}}=\frac{1}{2}<\alpha+\sigma .
$$

Thus, using the $X^{s, b}$ space embedding from Lemma 2.2, it suffices to prove

$$
\lim _{N \rightarrow \infty}\left\|w-w^{N}\right\|_{X_{\delta}^{\alpha+\sigma, \frac{1}{2}+}}=0
$$

where

$$
w^{N}:=\Phi_{t}^{N} f-e^{i t \Delta} \mathrm{P}_{\leq N} f^{\omega}, \quad w:=w^{\infty} .
$$

Notice that by (90) we have

$$
\left\|w^{N}\right\|_{X_{\delta}^{\alpha+\sigma, \frac{1}{2}+}}<1, \quad N \in 2^{\mathbb{N}} \cup\{\infty\} .
$$

Since for $t \in[0, \delta]$ we have

$$
w-w^{N}=-i \eta(t) \int_{0}^{t^{\prime}} e^{i\left(t-t^{\prime}\right) \Delta}\left(\mathcal{N}\left(\Phi_{t^{\prime}} f^{\omega}\right)-\mathrm{P}_{\leq N} \mathcal{N}\left(\Phi_{t^{\prime}}^{N} f^{\omega}\right)\right) d t^{\prime}
$$

using (28), (29), we get

$$
\left\|w-w^{N}\right\|_{X_{\delta}^{\alpha+\sigma, \frac{1}{2}+}} \lesssim \delta^{0+}\left\|\mathcal{N}\left(\Phi_{t} f\right)-\mathrm{P}_{\leq N} \mathcal{N}\left(\Phi_{t}^{N} f\right)\right\|_{X_{\delta}^{\alpha+\sigma,-\frac{1}{2}++}} .
$$

We decompose

$$
\begin{aligned}
& \mathcal{N}\left(\Phi_{t} f\right)-\mathrm{P}_{\leq N} \mathcal{N}\left(\Phi_{t}^{N} f\right)= \\
& \mathrm{P}_{\leq N}\left(\mathcal{N}\left(e^{i t \Delta} \mathrm{P}_{\leq N} f^{\omega}+w\right)-\mathcal{N}\left(e^{i t \Delta} \mathrm{P}_{\leq N} f^{\omega}+w^{N}\right)\right)+\text { Remainders }
\end{aligned}
$$

where

Remainders $:=\mathrm{P}_{\leq N}\left(\mathcal{N}\left(e^{i t \Delta} f^{\omega}+w\right)-\mathcal{N}\left(e^{i t \Delta} \mathrm{P}_{\leq N} f^{\omega}+w\right)\right)+\mathrm{P}_{>N} \mathcal{N}\left(\Phi_{t} f\right)$.

Notice that by (85), (87) we have

$$
\| \text { Remainders } \|_{X_{\delta}^{\alpha+\sigma,-\frac{1}{2}++}} \rightarrow 0 \text { as } N \rightarrow \infty \text {, }
$$

with probability at least $1-\varepsilon$. Using $(86)$ we can estimate

$$
\begin{aligned}
\| \mathrm{P}_{\leq N}\left(\mathcal{N}\left(e^{i t \Delta} \mathrm{P}_{\leq N} f^{\omega}+w\right)-\mathcal{N}\left(e^{i t \Delta} \mathrm{P}_{\leq N} f^{\omega}+\right.\right. & \left.\left.w_{N}\right)\right) \|_{X_{\delta}^{\alpha+\sigma,-\frac{1}{2}++}} \\
& \lesssim(-\ln \varepsilon)\left\|w-w^{N}\right\|_{X_{\delta}^{\alpha+\sigma, \frac{1}{2}+}}
\end{aligned}
$$

and (96), (97), (99) give

$$
\left\|w-w^{N}\right\|_{X_{\delta}^{\alpha+\sigma, \frac{1}{2}+}} \lesssim \delta^{0+}(-\ln \varepsilon)\left\|w-w^{N}\right\|_{X_{\delta}^{\alpha+\sigma, \frac{1}{2}+}}+\| \text { Remainders } \|_{X_{\delta}^{\alpha+\sigma,-\frac{1}{2}++}}
$$

with probability at least $1-\varepsilon$. Since with our choice of $\varepsilon=e^{-\delta^{-\gamma}}$ we have $C \delta^{0+}(-\ln \varepsilon)^{3 / 2}<1$, we can absorb the first term on the right hand side into the left hand side and we still have that (98) holds outside a $\delta$-exceptional set. Thus letting $N \rightarrow \infty$ the proof of (11) is complete.

To prove (12) we proceed as before. We show that for any $\delta>0$ sufficiently small we have

$$
\Phi_{t} f^{\omega}-f^{\omega} \in X_{\delta}^{\frac{d}{2}+, \frac{1}{2}+} .
$$


for $f^{\omega}$ outside a $\delta$-exceptional set $A_{\delta}$. This means that given $f^{\omega}$ we can find, $\mathbb{P}$ almost surely, a $\delta_{\omega}$ such that (101) is satisfied. Indeed, if we could not do so, this would mean that $f^{\omega} \in \bigcap_{\delta>0} A_{\delta}$, and the probability of this event is zero, since $\mathbb{P}\left(A_{\delta}\right) \rightarrow 0$ as $\delta \rightarrow 0$. Once we know that $\Phi_{t} f^{\omega}-e^{i t \Delta} f^{\omega} \in X_{\delta_{\omega}}^{\frac{d}{2}+, \frac{1}{2}+}$ we can use $X_{\delta_{\omega}}^{\frac{d}{2}+, \frac{1}{2}+} \hookrightarrow C_{t}\left(\left[0, \delta_{\omega}\right] ; H^{\frac{d}{2}+}\left(\Omega^{d}\right)\right)$ and $H^{\frac{d}{2}+}\left(\Omega^{d}\right) \hookrightarrow C_{x}\left(\Omega^{d}\right)$ (Sobolev embedding) to get (12), that so holds with probability $=1$. To prove (101) we use the smoothing, exactly as before, except that now we have to require the stronger inequality

$$
\frac{d}{2}<\alpha+\sigma .
$$

Since we can take $\sigma<\frac{1}{2}$ for $d=1,2$, we see that the previous condition is satisfied as long as $\alpha>\frac{d-1}{2}$. This concludes the proof.

Remark 4.8. It is worthy to remark that, comparing with for instance [5], the procedure which allows to promote a statement valid on a $\delta$-exceptional set $A_{\delta}$ for arbitrarily small $\delta>0$ to a statement which is valid with probability $=1$ is far easier. In particular we only need that $\lim _{\delta \rightarrow 0} \mathbb{P}\left(A_{\delta}\right)=0$ but we do not need any efficient upper bound of the convergence rate. This is because we are considering a property which has to be verified only at time $t=0$ a.s., instead that in a given small time interval containing $t=0$, as in [5].

We are now ready to prove the smoothing estimates given in Proposition 4.6.

Proof of Proposition 4.6. Notice that the Wick-ordered nonlinearity can be written as

$$
\mathcal{N}(u(x, \cdot))=\sum_{n_{2} \neq n_{1}, n_{3}} \widehat{u}\left(n_{1}\right) \widehat{\bar{u}}\left(n_{2}\right) \widehat{u}\left(n_{3}\right) e^{i\left(n_{1}-n_{2}+n_{3}\right) \cdot x}-\sum_{n} \widehat{u}(n)|\widehat{u}(n)|^{2} e^{i n \cdot x}
$$

where we are looking at the nonlinear term for fixed time and $\widehat{u}(\cdot)$ denotes the space Fourier coefficients. From (102), exploiting the symmetry $n_{1} \leftrightarrow n_{3}$, we also have the identity

$$
\begin{aligned}
& \mathcal{N}(u(x, \cdot))-\mathcal{N}(v(x, \cdot)) \\
& =\sum_{n_{2} \neq n_{1}, n_{3}}\left(\widehat{u}\left(n_{1}\right)-\widehat{v}\left(n_{1}\right)\right) \widehat{\bar{u}}\left(n_{2}\right) \widehat{u}\left(n_{3}\right) e^{i\left(n_{1}-n_{2}+n_{3}\right) \cdot x}-\sum_{n}(\widehat{u}(n)-\widehat{v}(n))|\widehat{u}(n)|^{2} e^{i n \cdot x} \\
& +\sum_{n_{2} \neq n_{1}, n_{3}}\left(\widehat{u}\left(n_{3}\right)-\widehat{v}\left(n_{3}\right)\right) \widehat{\bar{v}}\left(n_{2}\right) \widehat{v}\left(n_{1}\right) e^{i\left(n_{1}-n_{2}+n_{3}\right) \cdot x}-\sum_{n}(\widehat{u}(n)-\widehat{v}(n))|\widehat{v}(n)|^{2} e^{i n \cdot x} \\
& +\sum_{n_{2} \neq n_{1}, n_{3}}\left(\widehat{\bar{u}}\left(n_{2}\right)-\widehat{\bar{v}}\left(n_{2}\right)\right) \widehat{v}\left(n_{1}\right) \widehat{v}\left(n_{3}\right) e^{i\left(n_{1}-n_{2}+n_{3}\right) \cdot x}-\sum_{n}(\widehat{\bar{u}}(n)-\widehat{v}(n)) \widehat{u}(n) \widehat{v}(n) e^{i n \cdot x} .
\end{aligned}
$$

Using (103) (and recalling again the symmetry $n_{1} \leftrightarrow n_{3}$ ), it is clear that we can reduce to proving the (more general) Lemma 4.9 given below. It implies the desired statement since each summation in the above decomposition can be controlled by letting

$$
u_{j}\left(n_{j}\right)=u\left(n_{j}\right), v\left(n_{j}\right), \quad \text { or } u\left(n_{j}\right)-v\left(n_{j}\right) .
$$

The proof of Lemma 4.9 below follows closely the arguments introduced by Bourgain in [5]. We still display the details since we need to quantify the gain of 
regularity. One will note though that the proof of Lemma 4.9 reported here is much easier than the one presented in [5] since in our case $f^{\omega}$ is more regular, namely we consider $\alpha>0$ instead of $\alpha=0$.

Lemma 4.9. Let $d=1,2$ and $\alpha>0$. Let $N \in 2^{\mathbb{N}} \cup\{\infty\}$. For all $\sigma \in\left[0, \frac{1}{2}\right)$ the following holds. Assume for $j=1,2,3$

$$
u_{j}(I)=e^{i t \Delta} \mathrm{P}_{\leq N} f^{\omega}, \quad\left\|u_{j}(I I)\right\|_{X^{\alpha+\sigma, \frac{1}{2}+}}<1 .
$$

Let $J_{j} \in\{I, I I\}, j=1,2,3$. Then, for all $\varepsilon \in(0,1)$ sufficiently small we have the following

$$
\left\|\mathcal{N}\left(u_{1}\left(J_{1}\right), \overline{u_{2}}\left(J_{2}\right), u_{3}\left(J_{3}\right)\right)\right\|_{X^{\alpha+\sigma,-\frac{1}{2}+}} \lesssim(-\ln \varepsilon)^{3 / 2},
$$

and more precisely

$$
\begin{aligned}
& \left\|\mathcal{N}\left(u_{1}(I I), \overline{u_{2}}\left(J_{2}\right), u_{3}\left(J_{3}\right)\right)\right\|_{X^{\alpha+\sigma,-\frac{1}{2}++}} \lesssim(-\ln \varepsilon)\left\|u_{1}(I I)\right\|_{X^{\alpha+\sigma, \frac{1}{2}+}}, \\
& \left\|\mathcal{N}\left(u_{1}\left(J_{1}\right), \overline{u_{2}}(I I), u_{3}\left(J_{3}\right)\right)\right\|_{X^{\alpha+\sigma,-\frac{1}{2}++}} \lesssim(-\ln \varepsilon)\left\|u_{2}(I I)\right\|_{X^{\alpha+\sigma, \frac{1}{2}+}},
\end{aligned}
$$

with probability at least $1-\varepsilon$. Moreover, if in (104) we replace for some $j=j^{*}$ the projection operator $\mathrm{P}_{\leq N}$ by $\mathrm{P}_{>N}$, then the estimate (105) with $J_{j^{*}}=I$ holds with an extra factor $N^{-\alpha}$ on the right hand side.

Notice that by the symmetry $n_{1} \leftrightarrow n_{3}$ the estimate (106) implies an analogous estimate for $u_{3}(I I)$.

Before we pass to the proof we should remark that Lemma 4.9 proves an almost sure gain of smoothness of $\sigma=\frac{1}{2}-$ for the nonhomogeneous part of the solution of (9) with initial data $f^{\omega} \in H^{\alpha-}, \alpha>0$. This smoothing effect should be compared to the one recorded in Corollary 2.6 proved in a deterministic manner. There we proved that if the initial data is in $H^{0+}$ then basically there is only a $0++$ smoothing.

Proof. We prove (105), (106), (107) in the case $N=\infty$. It is then immediate to adapt the proof to $N \in \mathbb{N}$ and to prove the second part of the statement. Moreover, we first give the proof in dimension $d=2$, which is the hardest case. At the end of the proof we explain how to handle the case $d=1$. We split the nonlinearity into two parts:

$$
\begin{aligned}
& \mathcal{N}_{1}\left(u_{1}\left(J_{1}\right), \overline{u_{2}}\left(J_{2}\right), u_{3}\left(J_{3}\right)\right)=\sum_{n_{2} \neq n_{1}, n_{3}} \widehat{u_{1}\left(J_{1}\right)}\left(n_{1}\right) \widehat{\overline{u_{2}}\left(J_{2}\right)}\left(n_{2}\right) \widehat{u_{3}\left(J_{3}\right)}\left(n_{3}\right) e^{i\left(n_{1}-n_{2}+n_{3}\right) \cdot x}, \\
& \mathcal{N}_{2}\left(u_{1}\left(J_{1}\right), \overline{u_{2}}\left(J_{2}\right), u_{3}\left(J_{3}\right)\right)=\sum_{n} \widehat{u_{1}\left(J_{1}\right)}(n) \widehat{\overline{u_{2}\left(J_{2}\right)}}(n) \widehat{u_{3}\left(J_{2}\right)}(n) e^{i n \cdot x} .
\end{aligned}
$$

We prove (105), (106), (107) for $\mathcal{N}_{1}$, which is the most challenging contribution. The proof for $\mathcal{N}_{2}$ is elementary, so we leave the details to the reader. We decompose over dyadic scales $N_{1}, N_{2}, N_{3}$ in the following way:

$$
\begin{aligned}
& \left\|\mathcal{N}_{1}\left(u_{1}\left(J_{1}\right), \overline{u_{2}}\left(J_{2}\right), u_{3}\left(J_{3}\right)\right)\right\|_{X^{\alpha+\sigma,-\frac{1}{2}++}} \\
& \leq \sum_{N_{1}, N_{2}, N_{3}}\left\|\mathcal{N}_{1}\left(\mathrm{P}_{N_{1}} u_{1}\left(J_{1}\right), \mathrm{P}_{N_{2}} \overline{u_{2}}\left(J_{2}\right), \mathrm{P}_{N_{3}} u_{3}\left(J_{3}\right)\right)\right\|_{X^{\alpha+\sigma,-\frac{1}{2}++}} \\
& =: \sum_{M_{1}, M_{2}, M_{3}}\left\|\mathcal{N}_{1}\left(\mathrm{P}_{M_{1}} w_{1}\left(J_{1}\right), \mathrm{P}_{M_{2}} w_{2}\left(J_{2}\right), \mathrm{P}_{M_{3}} w_{3}\left(J_{3}\right)\right)\right\|_{X^{\alpha+\sigma,-\frac{1}{2}++}}
\end{aligned}
$$


where we denoted with $M_{1}, M_{2}, M_{3}$ the decreasing order of $N_{1}, N_{2}, N_{3}$. Notice that in this way $w_{1}$ denotes the $u_{j}$ supported on the largest frequency. We estimate this sum by first doing some reductions and then considering several cases. First we show that we can reduce to considering the case where the highest-frequency function is a random linear flow; i.e.

$$
w_{1}\left(J_{1}\right)=w_{1}(I) .
$$

Indeed if $w_{1}\left(J_{1}\right)=w_{1}(I I)$ we get, using (66)

$$
\begin{aligned}
& \left\|\mathcal{N}_{1}\left(\mathrm{P}_{M_{1}} w_{1}(I I), \mathrm{P}_{M_{2}} w_{2}\left(J_{2}\right), \mathrm{P}_{M_{3}} w_{3}\left(J_{3}\right)\right)\right\|_{X^{\alpha+\sigma,-\frac{1}{2}++}} \\
& \lesssim\left\|w_{1}(I I)\right\|_{X^{\alpha+\sigma, \frac{1}{2}+}}\left\|w_{2}\left(J_{2}\right)\right\|_{X^{0+, \frac{1}{2}+}}\left\|w_{3}\left(J_{3}\right)\right\|_{X^{0, \frac{1}{2}+}}
\end{aligned}
$$

On the other hand, recalling (104) and (83) we have

$$
\left\|w_{j}(I I)\right\|_{X^{\alpha+\sigma, \frac{1}{2}+}}<1, \quad\left\|w_{j}(I)\right\|_{X^{0+, \frac{1}{2}+}} \lesssim(-\ln \varepsilon)^{1 / 2},
$$

where the second inequality holds with probability at least $1-\varepsilon$. Thus, when $w_{1}\left(J_{1}\right)=w_{1}(I I)$ the estimates (105), (106), (107) follow summing the square of (109) over $M_{1}, M_{2}, M_{3}$, factorizing the sum, and then using Plancherel and (110). To prove the second bound in (110) one should notice that the space-time Fourier transform of $e^{i t \Delta} f^{\omega}$ is

$$
\widehat{e^{i t \Delta} f^{\omega}}(n, \tau)=\frac{g^{\omega}}{\langle n\rangle^{\frac{d}{2}+\alpha}} \delta\left(\tau+|n|^{2}\right),
$$

where $\delta$ is the delta function. So a direct computation gives

$$
\left\|e^{i t \Delta} f^{\omega}\right\|_{X^{0+, \frac{1}{2}+}}^{2}=\sum_{n} \frac{\left|g_{n}^{\omega}\right|^{2}}{\langle n\rangle^{d+2 \alpha-}}<\infty
$$

which using

$$
\int g_{n_{j}}^{\omega} g_{n_{j}^{\prime}}^{\omega} d \mathbb{P}(\omega)=0, \quad \int g_{n_{j}}^{\omega} \overline{g_{n_{j}^{\prime}}^{\omega}} d \mathbb{P}(\omega)=\left\{\begin{array}{lll}
0 & \text { if } & j \neq j^{\prime} \\
1 & \text { if } & j=j^{\prime}
\end{array},,\right.
$$

immediately implies

$$
\|\| e^{i t \Delta} f^{\omega}\left\|_{X^{0+, \frac{1}{2}+}}\right\|_{L_{\omega}^{2}}^{2} \sum_{n} \frac{1}{\langle n\rangle^{d+2 \alpha-}}<\infty .
$$

Using the hypercontractivity (basically (111) many times) we can upgrade this to an $L_{\omega}^{p}$ bound, for any $p<\infty$, with a constant $C p^{1 / 2}$ (see [23, Proposition 4.5] for details). Proceeding as in the proof of Lemma 4.3, this implies the second bound in (110) for all $\omega$ outside a set of probability smaller than $\varepsilon$, as required.

Then we perform a second reduction to remove frequencies which are far from the paraboloid. More precisely, we denote with $\mathrm{P}_{A}$ the space-time Fourier projection into the set $A$ and our goal is to reduce

$$
\begin{aligned}
& \sum_{M_{1}, M_{2}, M_{3}}\left\|\mathcal{N}_{1}\left(\mathrm{P}_{M_{1}} w_{1}(I), \mathrm{P}_{M_{2}} w_{2}\left(J_{2}\right), \mathrm{P}_{M_{3}} w_{3}\left(J_{3}\right)\right)\right\|_{X^{\alpha+\sigma,-\frac{1}{2}++}}^{2} \\
= & \sum_{N, M_{1}, M_{2}, M_{3}} N^{2 \alpha+2 \sigma}\left\|\mathrm{P}_{N} \mathcal{N}_{1}\left(\mathrm{P}_{M_{1}} w_{1}(I), \mathrm{P}_{M_{2}} w_{2}\left(J_{2}\right), \mathrm{P}_{M_{3}} w_{3}\left(J_{3}\right)\right)\right\|_{X^{0,-\frac{1}{2}++}}^{2}
\end{aligned}
$$


to

$\sum_{N, M_{1}, M_{2}, M_{3}} N^{2 \alpha+2 \sigma}\left\|\mathrm{P}_{N} \mathrm{P}_{\left\{\left\langle\tau+|n|^{2}\right\rangle \leq N^{1+\frac{1}{10}}\right\}} \mathcal{N}_{1}\left(\mathrm{P}_{M_{1}} w_{1}(I) \mathrm{P}_{M_{2}} w_{2}\left(J_{2}\right) \mathrm{P}_{M_{3}} w_{3}\left(J_{3}\right)\right)\right\|_{X^{0,-\frac{1}{2}++}}^{2}$

(the $\frac{1}{10}$ is removable, however it does not create any problems and facilitates the computations). To obtain this reduction, it is sufficient to show that projection of the nonlinearity onto the complementary set is appropriately bounded; i.e. that

$$
\begin{gathered}
\left.\sum_{N, M_{1}, M_{2}, M_{3}} N^{2 \alpha+2 \sigma} \| \mathrm{P}_{N} \mathrm{P}_{\left\{\left\langle\tau+|n|^{2}\right\rangle>N\right.} \frac{11}{10}\right\} \\
\mathcal{N}_{1}\left(\mathrm{P}_{M_{1}} w_{1}(I), \mathrm{P}_{M_{2}} w_{2}\left(J_{2}\right), \mathrm{P}_{M_{3}} w_{3}\left(J_{3}\right)\right) \|_{X^{0,-\frac{1}{2}++}}^{2} \\
\lesssim(-\ln \varepsilon)\left\|w_{2}\left(J_{2}\right)\right\|_{X^{0+, \frac{1}{2}+}}^{2}\left\|w_{3}\left(J_{2}\right)\right\|_{X^{0+, \frac{1}{2}+}}^{2}
\end{gathered}
$$

on a set of probability larger than $1-\varepsilon$. Indeed, recalling (110) and summing over $N$, this would imply the validity of (105), (106), (107) for this term. We could have required a weaker bound than (114), replacing the $X^{0+, \frac{1}{2}+}$ norm with an $X^{\alpha+\sigma, \frac{1}{2}+}$ norm if $J_{2}=I I$ and with a $\ln \frac{1}{\varepsilon}$ factor if $J_{2}=I$. However, we are able to prove the stronger estimate (114). To do so we bound

$$
\begin{aligned}
& \sum_{M_{1}, M_{2}, M_{3}} N^{2 \alpha+2 \sigma}\left\|\mathrm{P}_{N} \mathrm{P}_{\left\{\left\langle\tau+|n|^{2}\right\rangle>N N^{\frac{11}{10}}\right\}} \mathcal{N}_{1}\left(\mathrm{P}_{M_{1}} w_{1}(I), \mathrm{P}_{M_{2}} w_{2}\left(J_{2}\right), \mathrm{P}_{M_{3}} w_{3}\left(J_{3}\right)\right)\right\|_{X^{0,-\frac{1}{2}++}}^{2} \\
& \sim N^{2 \alpha+2 \sigma} \sum_{\substack{M_{1}, M_{2}, M_{3} \\
n \sim N}} \int \frac{\chi_{\left\{\left\langle\tau+|n|^{2}\right\rangle>N\right.}}{\left.\left\langle\tau+|n|^{2}\right\rangle^{1-}\right\}}\left|\widehat{\mathcal{N}_{1}(\cdot)}(n, \tau)\right|^{2} d \tau \\
& \lesssim N^{2 \alpha-} \sum_{\substack{M_{1}, M_{2}, M_{3} \\
n \sim N}} \int\left|\widehat{\mathcal{N}_{1}(\cdot)}(n, \tau)\right|^{2} d \tau \\
& \sim N^{2 \alpha-} \sum_{M_{1}, M_{2}, M_{3}}\left\|\mathrm{P}_{N} \mathcal{N}_{1}\left(\mathrm{P}_{M_{1}} w_{1}(I), \mathrm{P}_{M_{2}} w_{2}\left(J_{2}\right), \mathrm{P}_{M_{3}} w_{3}\left(J_{3}\right)\right)\right\|_{L_{x, t}^{2}}^{2} .
\end{aligned}
$$

Then using Hölder's inequality, the improved Strichartz inequality (73) for randomized functions (for the $L^{q}$ norm of $w_{1}(I)$ ), and the Strichartz inequality (23) (for the $L^{4}$ norms of $w_{2}\left(J_{2}\right)$ and $\left.w_{3}\left(J_{3}\right)\right)$, we obtain

$$
\begin{aligned}
\| \mathrm{P}_{N} & \mathcal{N}_{1}\left(\mathrm{P}_{M_{1}} w_{1}(I), \mathrm{P}_{M_{2}} w_{2}\left(J_{2}\right), \mathrm{P}_{M_{3}} w_{3}\left(J_{3}\right)\right) \|_{L_{x, t}^{2}}^{2} \\
& \leq\left\|\mathrm{P}_{M_{1}} w_{1}(I)\right\|_{L_{x, t}^{q}}^{2}\left\|\mathrm{P}_{M_{2}} w_{2}\left(J_{2}\right)\right\|_{L_{x, t}^{4+}}^{2}\left\|\mathrm{P}_{M_{3}} w_{3}\left(J_{3}\right)\right\|_{L_{x, t}^{4+}}^{2} \\
& \lesssim(-\ln \varepsilon) M_{1}^{-2 \alpha}\left\|\mathrm{P}_{M_{2}} w_{2}\left(J_{2}\right)\right\|_{L_{x, t}^{4+}}^{2}\left\|\mathrm{P}_{M_{3}} w_{3}\left(J_{3}\right)\right\|_{L_{x, t}^{4+}}^{2}, \\
& \lesssim(-\ln \varepsilon) M_{1}^{-2 \alpha}\left\|\mathrm{P}_{M_{2}} w_{2}\left(J_{2}\right)\right\|_{X^{0+, \frac{1}{2}}}^{2}\left\|\mathrm{P}_{M_{3}} w_{3}\left(J_{3}\right)\right\|_{X^{0+, \frac{1}{2}+}}^{2}
\end{aligned}
$$

where we are taking $q \gg 1$ sufficiently large. This holds on a set of probability larger than $1-\varepsilon$. Since $M_{1} \sim N$ once we plug (116) into into (115) the factor $N^{2 \alpha-}$ is absorbed by $M_{1}^{-2 \alpha}$ and we can rewrite the remaining factor as $M_{1}^{0-} N^{0-}$. Thus, summing over $N, M_{1}, M_{2}, M_{3}$ we obtain (114). So we have reduced to (113).

To handle this term we need a more explicit expression for the functions $w_{j}$. If we consider functions of the form $w(I)$ (here we omit the subscript $j$ to simplify 
the notation) we already know

$$
w(I)(x, t)=\sum_{m} \frac{g_{m}^{\omega}}{\langle m\rangle^{1+\alpha}} e^{i m \cdot x-i|m|^{2} t} .
$$

We can obtain a similar expression for $w(I I)$, namely

$$
w(I I)(x, t)=\int \phi(\lambda) \sum_{m} b_{\lambda}(m) e^{i m \cdot x-i|m|^{2} t} d \lambda,
$$

where $\phi$ satisfies

$$
\int|\phi(\lambda)| d \lambda \lesssim\|w(I I)\|_{X^{\alpha+\sigma, \frac{1}{2}+}},
$$

and the coefficients $b_{\lambda}(m)$ satisfy

$$
\sum_{m}\langle m\rangle^{2 \alpha+1-}\left|b_{\lambda}(m)\right|^{2}=1 .
$$

To prove (118)-(120) we change variables by setting $\tau^{\prime}=\tau+|m|^{2}$ :

$$
\begin{aligned}
& w(I I)(x, t)=\sum_{m} \int e^{i x \cdot m+i t \cdot \tau} \widehat{w(I I)}(m, \tau) d \tau \\
& =\sum_{m} \int e^{i t \cdot \tau^{\prime}} e^{i m \cdot x-i|m|^{2} t} \widehat{w(I I)}\left(m, \tau^{\prime}-|m|^{2}\right) d \tau^{\prime} \\
& =\int\left(\left.\sum_{\ell} \ell^{2 \alpha+2 \sigma} \widehat{\mid w(I I)}\left(\ell, \tau^{\prime}-|\ell|^{2}\right)\right|^{2}\right)^{\frac{1}{2}} e^{i t \cdot \tau^{\prime}} \sum_{m} e^{i m \cdot x-i|m|^{2} t} b_{\tau^{\prime}}(m) d \tau^{\prime},
\end{aligned}
$$

where we have defined

$$
b_{\lambda}(m):=\frac{\widehat{w(I I)}\left(m, \lambda-|m|^{2}\right)}{\left(\sum_{\ell}\langle\ell\rangle^{2 \alpha+2 \sigma}\left|\widehat{w(I I)}\left(\ell, \lambda-|\ell|^{2}\right)\right|^{2}\right)^{\frac{1}{2}}} .
$$

Thus (118) holds with

$$
\phi(\lambda):=\left(\left.\sum_{\ell}\langle\ell\rangle^{2 \alpha+2 \sigma} \widehat{\mid w(I I)}\left(\ell, \lambda-|\ell|^{2}\right)\right|^{2}\right)^{\frac{1}{2}} e^{i t \cdot \lambda}
$$

Notice that (120) is immediate by the definition (121). The property (119) follows by the Cauchy-Schwartz inequality and changing variables $\lambda^{\prime}=\lambda-|\ell|^{2}$ :

$$
\begin{aligned}
\int|\phi(\lambda)| d \lambda & \leq\left(\int \frac{d \lambda}{\langle\lambda\rangle^{1+}}\right)^{\frac{1}{2}}\left(\left.\langle\lambda\rangle^{1+}\langle\ell\rangle^{2 \alpha+2 \sigma} \widehat{\mid w(I I)}\left(\ell, \lambda-|\ell|^{2}\right)\right|^{2} d \lambda\right)^{\frac{1}{2}} \\
& \lesssim\left(\left\langle\lambda^{\prime}+|\ell|^{2}\right\rangle^{1+}\langle\ell\rangle^{2 \alpha+2 \sigma} \widehat{\mid w(I I)}\left(\ell, \lambda^{\prime}\right) d \lambda^{\prime}\right)^{\frac{1}{2}}=\|w(I I)\|_{X^{\alpha+\sigma, \frac{1}{2}+}}
\end{aligned}
$$

We now come back to the $u$ functions and introduce the notation

$$
a_{u(J), \lambda}(m):= \begin{cases}\frac{g_{m}^{\omega}}{\langle m\rangle^{1+\alpha}} & \text { if } J=I, \\ b_{\lambda}(m) & \text { if } J=I I .\end{cases}
$$

Recalling (117) and (118), we have

$$
\mathrm{P}_{N} \mathrm{P}_{\left\{\left\langle\tau+|n|^{2}\right\rangle \leq N^{2 s}\right\}} \mathcal{N}_{1}\left(\mathrm{P}_{N_{1}} u_{1}(I), \mathrm{P}_{N_{2}} u_{2}\left(J_{2}\right), \mathrm{P}_{N_{3}} u_{3}\left(J_{3}\right)\right)
$$




$$
\begin{gathered}
=\int \mathrm{P}_{N} \mathrm{P}_{\left\{\left\langle\tau+|n|^{2}\right\rangle \leq N^{2 s}\right\}}\left(\sum_{\left|n_{j}\right| \sim N_{j}} e^{i x \cdot\left(n_{1}-n_{2}+n_{3}\right)} e^{-i t\left(\left|n_{1}\right|^{2}-\left|n_{2}\right|^{2}+\left|n_{3}\right|^{2}\right)}\right) \\
\times \prod_{j=1,2,3} a_{u_{j}\left(J_{j}\right), \lambda_{j}}\left(n_{j}\right) \delta_{J_{j}}\left(\phi\left(\lambda_{j}\right) d \lambda_{j}\right),
\end{gathered}
$$

where

$$
\delta_{J_{j}}\left(\phi\left(\lambda_{j}\right) d \lambda_{j}\right)= \begin{cases}1 & \text { if } J_{j}=I \\ \phi\left(\lambda_{j}\right) d \lambda_{j} & \text { if } J_{j}=I I .\end{cases}
$$

Thus using Minkowski's inequality and recalling (119) we see that (113) satisfies the desired inequalities (105), (106), (107) as long as we can bound

$$
\begin{array}{r}
N^{2 \alpha+2 \sigma} \| \sum_{N_{1}, N_{2}, N_{3}} \mathrm{P}_{N} \mathrm{P}_{\left\{\left\langle\tau+|n|^{2}\right\rangle \leq N \frac{11}{10}\right\}}\left(\sum_{\left|n_{j}\right| \sim N_{j}} e^{i x \cdot\left(n_{1}-n_{2}+n_{3}\right)} e^{-i t\left(\left|n_{1}\right|^{2}-\left|n_{2}\right|^{2}+\left|n_{3}\right|^{2}\right)}\right) \\
\times \prod_{j=1,2,3} a_{u_{j}\left(J_{j}\right), \lambda_{j}} \|_{X^{0,-\frac{1}{2}++}}^{2} \lesssim(-\ln \varepsilon)^{3} N^{0-},
\end{array}
$$

uniformly in $\lambda_{j}$, for all $\varepsilon \in(0,1)$ sufficiently small, on a set of probability larger than $1-\varepsilon$. All the following estimates are indeed uniform in $\lambda_{j}$ and the exceptional set on which (124) could be not satisfied is independent of $\lambda_{j}$. We omit the subscript $\lambda_{j}$ to simplify the notation.

Since

$$
\begin{aligned}
\mathcal{F}\left(e^{i x \cdot\left(n_{1}-n_{2}+n_{3}\right)} e^{-i t\left(\left|n_{1}\right|^{2}-\left|n_{2}\right|^{2}+\left|n_{3}\right|^{2}\right)}\right)(n, \tau) & \\
& =\sum_{n_{1}-n_{2}+n_{3}=n} \delta\left(\tau+\left|n_{1}\right|^{2}-\left|n_{2}\right|^{2}+\left|n_{3}\right|^{2}\right),
\end{aligned}
$$

where $\mathcal{F}$ is the space-time Fourier transform and $\delta$ is the delta function, we reduce (124) to showing that

$$
\begin{aligned}
N^{2 \alpha+2 \sigma} \sum_{N_{1}, N_{2}, N_{3}} & \sum_{|n| \sim N} \frac{\left.\chi_{\left\{\left\langle|n|^{2}-\left|n_{1}\right|^{2}+\left|n_{2}\right|^{2}-\left|n_{3}\right|^{2}\right\rangle \leq N\right.} \frac{11}{10}\right\}}{\left\langle|n|^{2}-\left|n_{1}\right|^{2}+\left|n_{2}\right|^{2}-\left|n_{3}\right|^{2}\right\rangle^{1--}} \\
& \times\left|\sum_{\substack{\left|n_{j}\right| \sim N_{j}, n_{2} \neq n_{1}, n_{3} \\
n=n_{1}-n_{2}+n_{3}}} \prod_{j=1,2,3} a_{u_{j}\left(J_{j}\right)}\left(n_{j}\right)\right|^{2} \lesssim(-\ln \varepsilon)^{3} N^{0-} .
\end{aligned}
$$

Letting

$$
\mu=|n|^{2}-\left|n_{1}\right|^{2}+\left|n_{2}\right|^{2}-\left|n_{3}\right|^{2} .
$$

we see that (126) follows by

$$
\begin{array}{r}
N^{2 \alpha+2 \sigma} \sum_{N_{1}, N_{2}, N_{3}} \sum_{\mu \in \mathbb{Z},\langle\mu\rangle \leq N \frac{11}{10}} \frac{1}{\langle\mu\rangle^{1--}} \sum_{|n| \sim N}\left|\sum_{R_{n}\left(n_{1}, n_{2}, n_{3}\right)} \prod_{j=1,2,3} a_{u_{j}\left(J_{j}\right)}\left(n_{j}\right)\right|^{2} \\
\lesssim(-\ln \varepsilon)^{3} N^{0-}
\end{array}
$$


where for fixed $n, \mu$ we have denoted

$$
\begin{aligned}
R_{n}\left(n_{1}, n_{2}, n_{3}\right):= & \left\{\left(n_{1}, n_{2}, n_{3}\right) \in \mathbb{Z}^{3}:\left|n_{j}\right| \sim N_{j}, j=1,2,3,\right. \\
& \left.n_{2} \neq n_{1}, n_{3}, n_{1}-n_{2}+n_{3}=n, \mu=|n|^{2}-\left|n_{1}\right|^{2}+\left|n_{2}\right|^{2}-\left|n_{3}\right|^{2}\right\} .
\end{aligned}
$$

The set $R_{n}(\cdot)$ depends on $\mu$ also (like all the sets we define below). However we omit this dependence to simplify the notation. Since summing $\langle\mu\rangle^{-1+}$ gives an $N^{0+}$ factor, we reduced to prove

$$
\begin{aligned}
\left.N^{2 \alpha+2 \sigma} \sum_{N_{1}, N_{2}, N_{3}} \sup _{|\mu| \lesssim N} \sum_{\frac{11}{10}}\left|\sum_{|n| \sim N}\right| \prod_{R_{n}\left(n_{1}, n_{2}, n_{3}\right)} \prod_{j=1,2,3} a_{u_{j}\left(J_{j}\right)}\left(n_{j}\right)\right|^{2} \\
\lesssim(-\ln \varepsilon)^{3} N^{0-},
\end{aligned}
$$

Notice that in the definition of $R_{n}(\cdot)$ the condition

$$
|n|^{2}-\left|n_{1}\right|^{2}+\left|n_{2}\right|^{2}-\left|n_{3}\right|^{2}=\mu
$$

can be equivalently replaced by

$$
2\left(n_{1}-n_{2}\right) \cdot\left(n_{3}-n_{2}\right)=\mu \text {. }
$$

Recalling that $M_{1}, M_{2}, M_{3}$ is the decreasing order of $N_{1}, N_{2}, N_{3}$, we now notice that we must have $N_{1} \sim M_{1}$ or $N_{3} \sim M_{1}$. Indeed, if we assume that both $N_{1} \ll M_{1}$ and $N_{3} \ll M_{1}$ we must have $N_{2} \sim M_{1} \sim N$ and $\mu \sim N^{2}$, which contradicts the fact that $\mu \sim N^{\frac{11}{10}}$. Since the roles of $N_{1}$ and $N_{3}$ are symmetric (they are always the size of the indices of the Fourier coefficents of $u_{1}, u_{3}$ ), hereafter we assume that

$$
N_{1}=M_{1} \sim N \quad \text { and so } u_{1}=w_{1} ;
$$

recall that $w_{1}$ is the $u_{j}$ supported on the largest frequency, and we have previously reduced to considering $w_{1}\left(J_{1}\right)=w_{1}(I)$; see (108). Thus, the argument above allows us to further reduce (129) to showing that

$$
N^{2 \alpha+2 \sigma} \sum_{N_{1}, N_{2}, N_{3}} \sup _{|\mu| \lesssim N_{1}^{10}} \sum_{|n| \sim N_{1}}\left|\sum_{R_{n}\left(n_{1}, n_{2}, n_{3}\right)} \frac{g_{n_{1}}^{\omega}}{\left\langle n_{1}\right\rangle^{1+\alpha}} a_{u_{2}\left(J_{2}\right)}\left(n_{2}\right) a_{u_{3}\left(J_{3}\right)}\left(n_{3}\right)\right|^{2} \lesssim(-\ln \varepsilon)^{3} N^{0-} .
$$

To estimate (130) we can now distinguish few last possibilities. It is useful to denote

$$
\begin{aligned}
S\left(n_{1}, n_{2}, n_{3}\right):= & \left\{\left(n_{1}, n_{2}, n_{3}\right) \in \mathbb{Z}^{3}:\left|n_{j}\right| \sim N_{j}, j=1,2,3,\right. \\
& \left.n_{2} \neq n_{1}, n_{3}, \mu=2\left(n_{1}-n_{2}\right) \cdot\left(n_{3}-n_{2}\right)\right\} .
\end{aligned}
$$

Case $J_{2}=J_{3}=I$. We must show that

$$
N^{2 \alpha+2 \sigma} \sum_{N_{1}, N_{2}, N_{3}} \sup _{|\mu| \lesssim N_{1}^{\frac{11}{10}}} \sum_{|n| \sim N_{1}}\left|\sum_{R_{n}\left(n_{1}, n_{2}, n_{3}\right)} \frac{g_{n_{1}}^{\omega}}{\left\langle n_{1}\right\rangle^{1+\alpha}} \frac{\overline{g_{n_{2}}^{\omega}}}{\left\langle n_{2}\right\rangle^{1+\alpha}} \frac{g_{n_{3}}^{\omega}}{\left\langle n_{3}\right\rangle^{1+\alpha}}\right|^{2} \lesssim(-\ln \varepsilon)^{3} N^{0-} .
$$


Recalling that (111) along with the fact that the sum is restricted over $n_{1}, n_{3} \neq n_{2}$ and symmetric under $n_{1} \leftrightarrow n_{3}$, we get

$$
\int\left|\sum_{R_{n}\left(n_{1}, n_{2}, n_{3}\right)} \frac{g_{n_{1}}^{\omega}}{\left\langle n_{1}\right\rangle^{1+\alpha}} \frac{\overline{g_{n_{2}}^{\omega}}}{\left\langle n_{2}\right\rangle^{1+\alpha}} \frac{g_{n_{3}}^{\omega}}{\left\langle n_{3}\right\rangle^{1+\alpha}}\right|^{2} d \mathbb{P}(\omega)=2 \sum_{R_{n}\left(n_{1}, n_{2}, n_{3}\right)} \frac{1}{\left\langle n_{1}\right\rangle^{2 \alpha+2}} \frac{1}{\left\langle n_{2}\right\rangle^{2 \alpha+2}} \frac{1}{\left\langle n_{3}\right\rangle^{2 \alpha+2}} .
$$

In the following bound we first restrict the summation over $\left(n_{1}, n_{2}, n_{3}\right) \in R_{n}\left(n_{1}, n_{2}, n_{3}\right)$ such that $n_{1} \neq n_{3}$ (with a small abuse of notation we do not introduce additional notation for this restriction). In this case

$$
\begin{aligned}
& \int \sum_{|n| \sim N_{1}}\left|\sum_{R_{n}\left(n_{1}, n_{2}, n_{3}\right)} \frac{g_{n_{1}}^{\omega}}{\left\langle n_{1}\right\rangle^{1+\alpha}} \frac{\frac{g_{n_{2}}^{\omega}}{\left\langle n_{2}\right\rangle^{1+\alpha}}}{\left\langle n_{3}\right\rangle^{1+\alpha}}\right|^{2} d \mathbb{P}(\omega) \\
& \lesssim \sum_{|n| \sim N_{1}} \sum_{R_{n}\left(n_{1}, n_{2}, n_{3}\right)} \frac{1}{\left\langle n_{1}\right\rangle^{2 \alpha+2}} \frac{1}{\left\langle n_{2}\right\rangle^{2 \alpha+2}} \frac{1}{\left\langle n_{3}\right\rangle^{2 \alpha+2}} \\
& \lesssim \sum_{S\left(n_{1}, n_{2}, n_{3}\right)} \frac{1}{\left\langle n_{1}\right\rangle^{2 \alpha+2}} \frac{1}{\left\langle n_{2}\right\rangle^{2 \alpha+2}} \frac{1}{\left\langle n_{3}\right\rangle^{2 \alpha+2}} \\
& \sim \sum_{S\left(n_{1}, n_{2}, n_{3}\right)} N_{1}^{-2 \alpha-2} N_{2}^{-2 \alpha-2} N_{3}^{-2 \alpha-2} \\
& \lesssim N_{1}^{-2 \alpha-2} N_{2}^{-2 \alpha-2} N_{3}^{-2 \alpha-2} \# S\left(n_{1}, n_{2}, n_{3}\right) \\
& \lesssim N_{1}^{-2 \alpha-1} N_{2}^{-2 \alpha} N_{3}^{-2 \alpha},
\end{aligned}
$$

where we used that if $n_{1} \neq n_{3}$, then

$$
\# S\left(n_{1}, n_{2}, n_{3}\right) \lesssim N_{1} N_{2}^{2} N_{3}^{2}
$$

this is because once we have fixed $n_{2}, n_{3}$ in $N_{2}^{2} N_{3}^{2}$ possible ways, we remain with at most $N_{1}$ choices for $n_{1}$ by the relation $\mu=2\left(n_{1}-n_{2}\right) \cdot\left(n_{3}-n_{2}\right)$. If we sum over $\left(n_{1}, n_{2}, n_{3}\right) \in R_{n}\left(n_{1}, n_{2}, n_{3}\right)$ such that $n_{1}=n_{3}$, the restriction $\mu=2\left|n_{1}-n_{2}\right|^{2}$ implies that once we have chosen $n_{2}$ in $N_{2}^{2}$ possible ways, we remain with $\lesssim \mu^{0+} \lesssim$ $N_{1}^{0++}$ choices for $n_{1}=n_{3}$ (since a circle of radius $\mu$ contains $\lesssim \mu^{0+}$ integer points). This gives an even better bound than the one above. Summing the (133) over $N_{2}, N_{3}$ and recalling that $N_{1} \sim N$, we have bounded the $L_{\omega}^{2}$ norm of the left hand side of (132) by

$$
N^{2 \alpha+2 \sigma} \sum_{N_{1}} N_{1}^{-2 \alpha-1} \lesssim N^{2 \sigma-1} \lesssim N^{0-}
$$

where we used $\sigma<\frac{1}{2}$. Using the hypercontractivity of the Gaussian variables, we can upgrade this to an $L_{\omega}^{p}$ bound, for any $p<\infty$, with a constant $C p^{3 / 2}$ (see [23, Proposition 4.5] for details). Proceeding as in the proof of Lemma 4.3, this implies (132) for all $\omega$ outside a set of probability smaller than $\varepsilon$, as required.

Case $J_{2}=J_{3}=I I$. We show that

$$
N^{2 \alpha+2 \sigma} \sum_{N_{1}, N_{2}, N_{3}} \sup _{|\mu| \lesssim N_{1}^{\frac{11}{10}}} \sum_{|n| \sim N_{1}}\left|\sum_{R_{n}} \sum_{\left(n_{1}, n_{2}, n_{3}\right)} \frac{g_{n_{1}}^{\omega}}{\left\langle n_{1}\right\rangle^{1+\alpha}} b_{2}\left(n_{2}\right) b_{3}\left(n_{3}\right)\right|^{2} \lesssim(-\ln \varepsilon) N^{0-},
$$


which clearly implies (130). We denote

$$
R_{n, n_{2}}\left(n_{1}, n_{3}\right):=\left\{\left(n_{1}, n_{3}\right) \in \mathbb{Z}^{2}:\left(n_{1}, n_{2}, n_{3}\right) \in R_{n}\left(n_{1}, n_{2}, n_{3}\right)\right\},
$$

and for $j=2,3$

$$
\left\|b_{j}\right\|_{\ell_{N_{j}}^{2}}^{2}:=\sum_{\left|n_{j}\right| \sim N_{j}}\left|b_{j}\left(n_{j}\right)\right|^{2} .
$$

Notice that by (120) (and recalling the change in notations) we have for $\sigma<1 / 2$

$$
\sum_{N_{j}} N_{j}^{2 \alpha+2 \sigma}\left\|b_{j}\right\|_{\ell_{N_{j}}^{2}}^{2} \lesssim 1, \quad \sum_{N_{j} \lesssim N} N_{j}^{2 \alpha+1}\left\|b_{j}\right\|_{\ell_{N_{j}}^{2}}^{2} \lesssim N^{0+} .
$$

Hereafter all the sums over indexes $n_{j}$ are restricted to $n_{j} \sim N_{j}$. We omit this fact in the subscripts to simplify the notation. We estimate

$$
\begin{aligned}
\left.\sum_{R_{n}\left(n_{1}, n_{2}, n_{3}\right)} \frac{g_{n_{1}}^{\omega}}{\left\langle n_{1}\right\rangle^{1+\alpha}} b_{2}\left(n_{2}\right) b_{3}\left(n_{3}\right)\right|^{2} & \leq \sum_{n_{2}}\left|b_{2}\left(n_{2}\right)\right|^{2} \sum_{n_{2}}\left|\sum_{R_{n, n_{2}}\left(n_{1}, n_{3}\right)} \frac{g_{n_{1}}^{\omega}}{\left\langle n_{1}\right\rangle^{1+\alpha}} b_{3}\left(n_{3}\right)\right|^{2} \\
& \lesssim\left\|b_{2}\right\|_{\ell_{N_{2}}^{2}}^{2} \sum_{n_{2}}\left|\sum_{R_{n, n_{2}}\left(n_{1}, n_{3}\right)} \frac{g_{n_{1}}^{\omega}}{\left\langle n_{1}\right\rangle^{1+\alpha}} b_{3}\left(n_{3}\right)\right|^{2}
\end{aligned}
$$

where we have used the Cauchy-Schwartz inequality with respect to $n_{2}$ and (120). We further denote

$$
S_{n_{2}}\left(n_{1}, n_{3}\right):=\left\{\left(n_{1}, n_{3}\right) \in \mathbb{Z}^{2}:\left(n_{1}, n_{2}, n_{3}\right) \in S\left(n_{1}, n_{2}, n_{3}\right)\right\} .
$$

We recall the estimate

$$
\# S_{n_{2}}\left(n_{1}, n_{3}\right) \lesssim N_{1}^{0+} \quad \text { (Lemma } 1 \text { part (i) in [5]) . }
$$

Thus, summing the (136) over $|n| \sim N_{1}$ yields

$$
\begin{aligned}
& \sum_{n}\left|\sum_{R_{n}\left(n_{1}, n_{2}, n_{3}\right)} \frac{g_{n_{1}}^{\omega}}{\left\langle n_{1}\right\rangle^{1+\alpha}} b_{2}\left(n_{2}\right) b_{3}\left(n_{3}\right)\right|^{2} \\
& \lesssim\left\|b_{2}\right\|_{\ell_{N_{2}}^{2}}^{2} \sum_{n, n_{2}}\left|\sum_{R_{n, n_{2}}\left(n_{1}, n_{3}\right)} \frac{g_{n_{1}}^{\omega}}{\left\langle n_{1}\right\rangle^{1+\alpha}} b_{3}\left(n_{3}\right)\right|^{2} \\
& \lesssim(-\ln \varepsilon)\left\|b_{2}\right\|_{\ell_{N_{2}}^{2}}^{2} \sum_{n_{2}}\left(\sum_{S_{n_{2}}\left(n_{1}, n_{3}\right)} \frac{\left|b_{3}\left(n_{3}\right)\right|}{\left\langle n_{1}\right\rangle^{1+\alpha}}\right)^{2} \\
& \lesssim(-\ln \varepsilon)\left\|b_{2}\right\|_{\ell_{N_{2}}^{2}}^{2} N_{1}^{0+} \sum_{n_{2}} \sum_{S_{n_{2}}\left(n_{1}, n_{3}\right)} \frac{\left|b_{3}\left(n_{3}\right)\right|^{2}}{\left\langle n_{1}\right\rangle^{2 \alpha+2}} \\
& \lesssim(-\ln \varepsilon)\left\|b_{2}\right\|_{\ell_{N_{2}}^{2}}^{2} N_{1}^{0+} N_{1}^{-2 \alpha-2} \sum_{S\left(n_{1}, n_{2}, n_{3}\right)}\left|b_{3}\left(n_{3}\right)\right|^{2},
\end{aligned}
$$

where we used (137) and the fact that

$$
\sum_{S\left(n_{1}, n_{2}, n_{3}\right)}=\sum_{n_{2}} \sum_{S_{n_{2}}\left(n_{1}, n_{3}\right)}
$$


To justify the previous computation, in particular the factor of $(-\ln \varepsilon)$, we should first average over $d \mathbb{P}(\omega)$ and then use the hypercontractivity of the Gaussian variables. Since this works exactly as in the previous case $\left(J_{2}=J_{3}=I\right)$, we omit the details. We do the same in (142). Denoting

$$
S_{n_{3}}\left(n_{1}, n_{2}\right):=\left\{\left(n_{1}, n_{2}\right) \in \mathbb{Z}^{3}:\left(n_{1}, n_{2}, n_{3}\right) \in S\left(n_{1}, n_{2}, n_{3}\right)\right\},
$$

we recall that

$$
\left.\# S_{n_{3}}\left(n_{1}, n_{2}\right) \lesssim N_{1}^{1+} N_{2} \quad \text { (Lemma } 2 \text { part (i) in [5] switching } n_{1} \text { and } n_{3}\right) \text {. }
$$

Since

$$
\sum_{S\left(n_{1}, n_{2}, n_{3}\right)}=\sum_{n_{3}} \sum_{S_{n_{3}}\left(n_{1}, n_{2}\right)}
$$

we have by (139)

$$
\sum_{S\left(n_{1}, n_{2}, n_{3}\right)}\left|b_{3}\left(n_{3}\right)\right|^{2} \lesssim N_{1}^{1+} N_{2} \sum_{n_{3}}\left|b_{3}\left(n_{3}\right)\right|^{2} \lesssim N_{1}^{1+} N_{2}\left\|b_{3}\right\|_{\ell_{N_{3}}^{2}}^{2} .
$$

Plugging (140) into (138) we see that (134) is satisfied as long as

$$
N^{2 \alpha+2 \sigma} \sum_{N_{1}, N_{2}, N_{3}} N_{1}^{-2 \alpha-1+0+} N_{2}\left\|b_{2}\right\|_{\ell_{N_{2}}^{2}}^{2}\left\|b_{3}\right\|_{\ell_{N_{3}}^{2}}^{2} \lesssim N^{0-} .
$$

Recalling (135) and the fact that $N \sim N_{1} \gtrsim N_{j}, j=2,3$, this is immediately verified for $\sigma<\frac{1}{2}$.

Case $J_{2}=I, J_{3}=I I$. We show that

$$
N^{2 \alpha+2 \sigma} \sum_{N_{1}, N_{2}, N_{3}} \sup _{|\mu| \lesssim N_{1}^{110}} \sum_{|n| \sim N_{1}}\left|\sum_{R_{n}\left(n_{1}, n_{2}, n_{3}\right)} \frac{g_{n_{1}}^{\omega}}{\left\langle n_{1}\right\rangle^{1+\alpha}} \frac{g_{n_{2}}^{\omega}}{\left\langle n_{2}\right\rangle^{1+\alpha}} b_{3}\left(n_{3}\right)\right|^{2} \lesssim(-\ln \varepsilon)^{2} N^{0-},
$$

which clearly implies (130). Since

$$
\# R_{n}\left(n_{1}, n_{2}, n_{3}\right) \lesssim N_{2} N_{3}^{0+} \quad \text { (Lemma } 1 \text { in [5]), }
$$

we can estimate using the Cauchy-Schwartz inequality:

$$
\begin{aligned}
& \left|\sum_{R_{n}\left(n_{1}, n_{2}, n_{3}\right)} \frac{g_{n_{1}}^{\omega}}{\left\langle n_{1}\right\rangle^{1+\alpha}} \frac{g_{n_{2}}^{\omega}}{\left\langle n_{2}\right\rangle^{1+\alpha}} b_{3}\left(n_{3}\right)\right|^{2} \\
& \lesssim(-\ln \varepsilon)^{2} N_{2} N_{3}^{0+} \sum_{R_{n}\left(n_{1}, n_{2}, n_{3}\right)} \frac{\left|b_{3}\left(n_{3}\right)\right|^{2}}{\left\langle n_{1}\right\rangle^{2+2 \alpha}\left\langle n_{2}\right\rangle^{2+2 \alpha}} \\
& \lesssim(-\ln \varepsilon)^{2} N_{2} N_{3}^{0+} N_{1}^{-2-2 \alpha} N_{2}^{-2-2 \alpha} \sum_{R_{n}\left(n_{1}, n_{2}, n_{3}\right)}\left|b_{3}\left(n_{3}\right)\right|^{2} \\
& \lesssim(-\ln \varepsilon)^{2} N_{1}^{-2-2 \alpha} N_{2}^{-1-2 \alpha} N_{3}^{0+} \sum_{R_{n}\left(n_{1}, n_{2}, n_{3}\right)}\left|b_{3}\left(n_{3}\right)\right|^{2} .
\end{aligned}
$$

Summing this over $\left|n_{1}\right| \sim N_{1}$ yields

$$
\sum_{\left|n_{1}\right| \sim N_{1}}\left|\sum_{R_{n}\left(n_{1}, n_{2}, n_{3}\right)} \frac{g_{n_{1}}^{\omega}}{\left\langle n_{1}\right\rangle^{1+\alpha}} \frac{g_{n_{2}}^{\omega}}{\left\langle n_{2}\right\rangle^{1+\alpha}} b_{3}\left(n_{3}\right)\right|^{2}
$$




$$
\begin{aligned}
& \lesssim(-\ln \varepsilon)^{2} N_{1}^{-2-2 \alpha} N_{2}^{-1-2 \alpha} N_{3}^{0+} \sum_{\left|n_{1}\right| \sim N_{1}} \sum_{R_{n}\left(n_{1}, n_{2}, n_{3}\right)}\left|b_{3}\left(n_{3}\right)\right|^{2} \\
& \lesssim(-\ln \varepsilon)^{2} N_{1}^{-2-2 \alpha} N_{2}^{-1-2 \alpha} N_{3}^{0+} \sum_{S\left(n_{1}, n_{2}, n_{3}\right)}\left|b_{3}\left(n_{3}\right)\right|^{2} .
\end{aligned}
$$

Then since

$$
\sum_{S\left(n_{1}, n_{2}, n_{3}\right)}=\sum_{n_{3}} \sum_{S_{n_{3}}\left(n_{1}, n_{2}\right)}
$$

and

$$
\# S_{n_{3}}\left(n_{1}, n_{2}\right) \lesssim N_{1}^{1+} N_{2}, \quad \text { (Lemma } 2 \text { part (i) in [5]) }
$$

we have

$$
\sum_{S\left(n_{1}, n_{2}, n_{3}\right)}\left|b_{3}\left(n_{3}\right)\right|^{2} N_{1}^{1+} N_{2} \sum_{n_{3}}\left|b_{3}\left(n_{3}\right)\right|^{2} \lesssim N_{1}^{1+} N_{2}\left\|b_{3}\right\|_{\ell_{N_{3}}^{3}}^{2} .
$$

Plugging (145) into (143) we see that the left hand side of (141) is bounded by

$$
N^{2 \alpha+2 \sigma} \sum_{N_{1}, N_{2}, N_{3}} N_{1}^{-2 \alpha-1+0+} N_{2}^{-2 \alpha} N_{3}^{0+}\left\|b_{3}\right\|_{\ell_{N_{3}}^{3}}^{2} \lesssim N^{0-}
$$

as required, where we used $\sigma<\frac{1}{2}$, (135), and the fact that $N \sim N_{1} \gtrsim N_{3}$.

Case $J_{2}=I I, J_{3}=I$. We proceed exactly as in the case $J_{2}=I, J_{3}=I I$, but we exchange the roles of $n_{2}$ and $n_{3}$. Notice that everything works symmetrically under $n_{2} \leftrightarrow n_{3}$ except the fact that the sets $S_{n_{3}}\left(n_{1}, n_{2}\right)$ and $S_{n_{2}}\left(n_{1}, n_{3}\right)$ do not coincide. However, in the previous argument, we only needed the estimate (144). Here we instead use

$$
\# S_{n_{2}}\left(n_{1}, n_{3}\right) \lesssim N_{1}^{1+} N_{3}, \quad \text { (Lemma } 2 \text { part (ii) in [5]) }
$$

whose right hand side is indeed the same as that of (144) after interchanging $N_{2} \leftrightarrow$ $N_{3}$. This concludes the proof of (105), (106), (107) in dimension $d=2$.

The case $d=1$ is much easier. One can easily check that the previous argument indeed adapts and simplifies.

The quintic $N L S$ on $\mathbb{T}$. Here we explain how one can prove an analogous $\frac{1}{2}-$ smoothing result for the quintic NLS $(p=5)$ on $\mathbb{T}$, after removing certain bad resonances from the nonlinearity, as we have done using the Wick order in the cubic case. We plan to study this problem in detail in a future work. We consider ${ }^{8}$

$$
\mathcal{N}(u):= \pm u\left(|u|^{5}-3 \mu\right), \quad \mu=f_{\mathbb{T}}|u(x, t)|^{4} d x,
$$

and

$$
\left\{\begin{array}{l}
i \partial_{t} u+\Delta u=\mathcal{N}(u), \quad x \in \mathbb{T}, \\
u(x, 0)=f^{\omega}(x),
\end{array}\right.
$$

with randomized initial data

$$
f^{\omega}(x)=\sum_{n \in \mathbb{Z}} \frac{g_{n}^{\omega}}{\langle n\rangle^{\frac{1}{2}+\alpha}} e^{i n \cdot x} .
$$

\footnotetext{
${ }^{8}$ For more information about why this is the relevant nonlinear term to consider in the quintic case, consult [34].
} 
Recall that such data is $\mathbb{P}$-almost surely in $H^{s}$ for all $s<\alpha$ (namely we work at $H^{0+}$ level) and satisfies a uniform bound for these $H^{s}$ norms with arbitrarily high probability; see (83). Proceeding as for the cubic equation before, and focusing only on the fully random evolution, namely the case $J_{j}=I$ for $j=1, \ldots, 5$, we reduce to proving the following fact. We fix

$$
0<\sigma<\frac{1}{2}
$$

Then with probability at least $1-\varepsilon$, we have

$$
\begin{aligned}
N^{2 \alpha+2 \sigma} \sum_{N_{1}, \ldots, N_{5}} \sup _{\mu} \sum_{|n| \sim N_{1}} \mid \sum_{R_{n}\left(n_{1}, \ldots, n_{5}\right)} \frac{\frac{g_{n_{1}}^{\omega}}{\left\langle n_{1}\right\rangle^{\frac{1}{2}+\alpha}} \frac{\overline{g_{n_{2}}^{\omega}}}{\left\langle n_{2}\right\rangle^{\frac{1}{2}+\alpha}} \frac{g_{n_{3}}^{\omega}}{\left\langle n_{3}\right\rangle^{\frac{1}{2}+\alpha}}}{} \\
\times\left.\frac{\overline{g_{n_{4}}^{\omega}}}{\left\langle n_{4}\right\rangle^{\frac{1}{2}+\alpha}} \frac{g_{n_{5}}^{\omega}}{\left\langle n_{5}\right\rangle^{\frac{1}{2}+\alpha}}\right|^{2} \lesssim(-\ln \varepsilon)^{5} N^{0-}
\end{aligned}
$$

where

$$
\begin{aligned}
R_{n}\left(n_{1}, \ldots, n_{5}\right):= & \left\{\left(n_{1}, \ldots, n_{5}\right) \in \mathbb{Z}^{3}:\left|n_{j}\right| \sim N_{j}, j=1, \ldots, 5,\right. \\
& n_{2}, n_{4} \neq n_{1}, n_{3}, n_{5}, n_{1}-n_{2}+n_{3}-n_{4}+n_{5}=n, \\
& \left.\mu=|n|^{2}-\left|n_{1}\right|^{2}+\left|n_{2}\right|^{2}-\left|n_{3}\right|^{2}+\left|n_{4}\right|^{2}-\left|n_{5}\right|^{2}\right\}
\end{aligned}
$$

and we have assumed $N_{1}=\max \left\{N_{1}, \ldots, N_{5}\right\}$, so that we can restrict to the case $N \sim N_{1}$. However, the argument below adapts immediately to the case in which the largest frequency is $N_{2}$ (all the other cases are clearly symmetric). Again, averaging in $d \mathbb{P}(\omega)$ and upgrading the corresponding estimate to any $L_{\omega}^{p}, p \in[2, \infty)$ by hypercontractivity, we reduce to proving, uniformly over $\mu$, the following:

$$
N^{2 \alpha+2 \sigma} \sum_{N_{1}, \ldots, N_{5}} \sum_{|n| \sim N_{1}} \sum_{R_{n}} \prod_{\left(n_{1}, \ldots, n_{5}\right)} \frac{1}{j=1, \ldots, 5} \frac{1}{\left\langle n_{j}\right\rangle^{1+2 \alpha}} \lesssim N^{0-} .
$$

In fact, we have

$$
\begin{aligned}
& N^{2 \alpha+2 \sigma} \sum_{N_{1}, \ldots, N_{5}} \sum_{|n| \sim N_{1}} \sum_{R_{n}\left(n_{1}, \ldots, n_{5}\right)} \prod_{j=1, \ldots, 5} \frac{1}{\left\langle n_{j}\right\rangle^{1+2 \alpha}} \\
& \lesssim N^{2 \alpha+2 \sigma} \sum_{N_{1}, \ldots, N_{5}} \# R\left(n_{1}, \ldots, n_{5}\right) N_{1}^{-(1+2 \alpha)} N_{2}^{-(1+2 \alpha)} N_{3}^{-(1+2 \alpha)} N_{4}^{-(1+2 \alpha)} N_{5}^{-(1+2 \alpha)}
\end{aligned}
$$

and since $\# R\left(n_{1}, \ldots, n_{5}\right) \lesssim N_{5} N_{4} N_{3} N_{2}$ and $\sigma<\frac{1}{2}$, the estimate (151) is proved.

\subsection{The Cubic NLS Equation on $\mathbb{R}^{d}(d=1,2)$ with Random Data (The- orem 1.5).}

We prove Theorem 1.5. Given $f \in H^{s}\left(\mathbb{R}^{d}\right)$ with $s>0$, we are considering the randomized initial data $f^{\omega}$ defined in (14). Remember that these functions are typically more integrable than $f$. They are $\mathbb{P}$-almost surely in $L^{p}$ for any $p \in[2, \infty)$. On the other hand, they are not more regular than $f$, but they rather have comparable $H^{s}$ norms; see (82). We approximate the equation (here $\mathcal{N}(z)=$ $\left.\pm|z|^{2} z\right)$ as in (42), for all $N \in 2^{\mathbb{N}} \cup\{\infty\}$, and $\Phi_{t}^{N} f^{\omega}$ denotes the associated flow, with initial datum $f^{\omega}$. 
Proof of Theorem 1.5. Notice that (16) is the content of Proposition 4.5. To prove (17), proceeding exactly as in the proof of Theorem 1.3, it suffices that given any $\delta>0$ sufficiently small we prove

$$
\lim _{N \rightarrow \infty}\left\|\sup _{0 \leq t \leq \delta}\left|\Phi_{t} f^{\omega}(x)-\Phi_{t}^{N} f^{\omega}(x)\right|\right\|_{L_{x}^{2}\left(\mathbb{T}^{2}\right)}=0
$$

for all $f^{\omega}$ outside a $\delta$-exceptional set $A_{\delta}$. This can be done using Corollary 2.6 exactly as in the proof of (91) using Proposition 4.6. In fact, since Corollary 2.6 is a deterministic statement, we can actually prove (152) for all $\omega$. To do so we need to require (compare with (93))

$$
s_{\mathbb{R}^{d}}=\frac{d}{2(d+1)}<s+\sigma, \quad d=1,2 .
$$

Since we used Corollary 2.6, we are allowed to take $\sigma<\min (2 s, 1)$ and we see that (153) is satisfied for all $s>\frac{d}{6(d+1)}$. This completes the proof.

\section{REFERENCES}

[1] J. A. Barceló, J. Bennett, A. Carbery, and K. M. Rogers. On the dimension of divergence sets of dispersive equations. Math. Ann., 349(3):599-622, 2011.

[2] A. Bényi, T. Oh, and O. Pocovnicu. Wiener randomization on unbounded domains and an application to almost sure well-posedness of NLS. In Excursions in harmonic analysis. Vol. 4, Appl. Numer. Harmon. Anal., pages 3-25. Birkhäuser/Springer, Cham, 2015.

[3] J. Bourgain. A remark on Schrödinger operators. Israel J. Math., 77(1-2):1-16, 1992.

[4] J. Bourgain. Fourier transform restriction phenomena for certain lattice subsets and applications to nonlinear evolution equations. I. Schrödinger equations. Geom. Funct. Anal., 3(2):107-156, 1993.

[5] J. Bourgain. Invariant measures for the 2d-defocusing nonlinear Schrödinger equation. Communications in Mathematical Physics, 176(2):421-445, Mar 1996.

[6] J. Bourgain. On the Schrödinger maximal function in higher dimension. Tr. Mat. Inst. Steklova, 280(Ortogonal'nye Ryady, Teoriya Priblizheniǔ i Smezhnye Voprosy):53-66, 2013.

[7] J. Bourgain. A note on the Schrödinger maximal function. J. Anal. Math., 130:393-396, 2016.

[8] J. Bourgain and C. Demeter. The proof of the $l^{2}$ decoupling conjecture. Ann. of Math. (2), 182(1):351-389, 2015.

[9] N. Burq and N. Tzvetkov. Random data Cauchy theory for supercritical wave equations. I. Local theory. Invent. Math., 173(3):449-475, 2008.

[10] A. Carbery. Radial Fourier multipliers and associated maximal functions. In Recent progress in Fourier analysis (El Escorial, 1983), volume 111 of North-Holland Math. Stud., pages 49-56. North-Holland, Amsterdam, 1985.

[11] L. Carleson. Some analytic problems related to statistical mechanics. In Euclidean harmonic analysis (Proc. Sem., Univ. Maryland, College Park, Md., 1979), volume 779 of Lecture Notes in Math., pages 5-45. Springer, Berlin, 1980.

[12] J. E. Colliander, J.-M. Delort, C. E. Kenig, and G. Staffilani. Bilinear estimates and applications to 2D NLS. Trans. Amer. Math. Soc., 353(8):3307-3325, 2001.

[13] E. Compaan. A smoothing estimate for the nonlinear Schrödinger equation. UIUC Research Experiences for Graduate Students report, 2013.

[14] M. G. Cowling. Pointwise behavior of solutions to Schrödinger equations. In Harmonic analysis (Cortona, 1982), volume 992 of Lecture Notes in Math., pages 83-90. Springer, Berlin, 1983.

[15] B. E. J. Dahlberg and C. E. Kenig. A note on the almost everywhere behavior of solutions to the Schrödinger equation. In Harmonic analysis (Minneapolis, Minn., 1981), volume 908 of Lecture Notes in Math., pages 205-209. Springer, Berlin-New York, 1982.

[16] C. Demeter and S. Guo. Schrödinger maximal function estimates via the pseudoconformal transformation, 2016. 
[17] Y. Deng and P. Germain. Growth of solutions to NLS on irrational tori. Int. Math. Res. Not. IMRN, (9):2919-2950, 2019.

[18] Du and Zhang. Sharp $l^{2}$ estimates of the Schrödinger maximal function in higher dimensions. Ann. of Math., 189(3):837, 2019.

[19] X. Du, L. Guth, and X. Li. A sharp Schrödinger maximal estimate in $\mathbb{R}^{2}$. Ann. of Math., 186(2):607-640, 2017.

[20] X. Du, L. Guth, X. Li, and R. Zhang. Pointwise convergence of Schrödinger solutions and multilinear refined Strichartz estimates. Forum Math. Sigma, 6:e14, 18, 2018.

[21] M. Erdoğan and N. Tzirakis. Talbot effect for the cubic non-linear Schrödinger equation on the torus. Mathematical Research Letters, 20, 032013.

[22] M. B. Erdoğan and N. Tzirakis. Dispersive Partial Differential Equations: Wellposedness and Applications. London Mathematical Society Student Texts. Cambridge University Press, 2016.

[23] G. Genovese, R. Lucà, and D. Valeri. Gibbs measures associated to the integrals of motion of the periodic derivative nonlinear Schrödinger equation. Selecta Math. (N.S.), 22(3):1663$1702,2016$.

[24] T. Kato. On nonlinear Schrödinger equations. II. $H^{s}$-solutions and unconditional wellposedness. J. Anal. Math., 67:281-306, 1995.

[25] C. E. Kenig, G. Ponce, and L. Vega. Quadratic forms for the 1-D semilinear Schrödinger equation. Trans. Amer. Math. Soc., 348(8):3323-3353, 1996.

[26] R. Killip and M. Vişan. Scale invariant Strichartz estimates on tori and applications. Math. Res. Lett., 23(2):445-472, 2016.

[27] S. Lee. On pointwise convergence of the solutions to Schrödinger equations in $\mathbb{R}^{2}$. Int. Math. Res. Not., pages Art. ID 32597, 21, 2006.

[28] R. Lucà and K. Rogers. Average decay of the fourier transform of measures with applications. Journal of the European Mathematical Society, 21(2):465-506, Oct. 2018.

[29] R. Lucà and K. M. Rogers. Coherence on fractals versus pointwise convergence for the Schrödinger equation. Comm. Math. Phys., 351(1):341-359, 2017.

[30] R. Lucà and K. M. Rogers. A note on pointwise convergence for the Schrödinger equation. Math. Proc. Cambridge Philos. Soc., 166(2):209-218, 2019.

[31] J. Lührmann and D. Mendelson. Random data Cauchy theory for nonlinear wave equations of power-type on $\mathbb{R}^{3}$. Comm. Partial Differential Equations, 39(12):2262-2283, 2014.

[32] A. Moyua, A. Vargas, and L. Vega. Restriction theorems and maximal operators related to oscillatory integrals in $\mathbf{R}^{3}$. Duke Math. J., 96(3):547-574, 1999.

[33] A. Moyua and L. Vega. Bounds for the maximal function associated to periodic solutions of one-dimensional dispersive equations. Bull. Lond. Math. Soc., 40(1):117-128, 2008.

[34] A. R. Nahmod and G. Staffilani. Almost sure well-posedness for the periodic 3D quintic nonlinear Schrödinger equation below the energy space. J. Eur. Math. Soc. (JEMS), 17(7):1687$1759,2015$.

[35] E. M. Nikišin. A resonance theorem and series in eigenfunctions of the Laplace operator. Izv. Akad. Nauk SSSR Ser. Mat., 36:795-813, 1972.

[36] T. Oh, M. Okamoto, and O. Pocovnicu. On the probabilistic well-posedness of the nonlinear Schrödinger equations with non-algebraic nonlinearities. arXiv preprint arXiv:1708.01568, 2017.

[37] K. M. Rogers and P. Villarroya. Global estimates for the Schrödinger maximal operator. Ann. Acad. Sci. Fenn. Math., 32(2):425-435, 2007.

[38] P. Sjögren and P. Sjölin. Convergence properties for the time-dependent Schrödinger equation. Ann. Acad. Sci. Fenn. Ser. A I Math., 14(1):13-25, 1989.

[39] P. Sjölin. Regularity of solutions to the Schrödinger equation. Duke Math. J., 55(3):699-715, 1987.

[40] E. M. Stein. On limits of sequences of operators. Ann. of Math., 74(1):140-170, 1961.

[41] R. S. Strichartz. Restrictions of Fourier transforms to quadratic surfaces and decay of solutions of wave equations. Duke Math. J., 44(3):705-714, 1977.

[42] T. Tao. Multilinear weighted convolution of $L^{2}$-functions, and applications to nonlinear dispersive equations. Amer. J. Math., 123(5):839-908, 2001.

[43] T. Tao. A sharp bilinear restrictions estimate for paraboloids. Geom. Funct. Anal., 13(6):1359-1384, 2003. 
[44] T. Tao and C. B. of the Mathematical Sciences. Nonlinear Dispersive Equations: Local and Global Analysis. Conference Board of the Mathematical Sciences. Regional conference series in mathematics. American Mathematical Society, 2006.

[45] T. Tao and A. Vargas. A bilinear approach to cone multipliers. I. Restriction estimates. Geom. Funct. Anal., 10(1):185-215, 2000.

[46] T. Tao and A. Vargas. A bilinear approach to cone multipliers. II. Applications. Geom. Funct. Anal., 10(1):216-258, 2000.

[47] L. Vega. Schrödinger equations: pointwise convergence to the initial data. Proc. Amer. Math. Soc., 102(4):874-878, 1988.

[48] X. Wang and C. Zhang. Pointwise convergence of solutions to the schrödinger equation on manifolds. Canadian Journal of Mathematics, 71(4):983-995, 2019.

[49] T. Zhang and D. Fang. Random data Cauchy theory for the generalized incompressible Navier-Stokes equations. J. Math. Fluid Mech., 14(2):311-324, 2012.

Department of Mathematics, Massachusetts Institute of Technology

Email address: compaan@mit.edu

BCAM - Basque Center for Applied Mathematics, 48009 Bilbao, Spain and Ikerbasque, Basque Foundation for Science, 48011 Bilbao, Spain.

Email address: rluca@bcamath.org

Department of Mathematics, Massachusetts Institute of Technology

Email address: gigliola@math.mit.edu 